Cite this: RSC Adv., 2018, 8, 11813

Received 30th December 2017 Accepted 3rd March 2018

DOI: 10.1039/c7ra13760e

rsc.li/rsc-advances
Check for updates

\section{Identification of metabolites of liquiritin in rats by UHPLC-Q-TOF-MS/MS: metabolic profiling and pathway comparison in vitro and in vivo $\dagger$}

\begin{abstract}
Xia Zhang, Caijuan Liang, Jintuo Yin, Yupeng Sun and Lantong Zhang (D)*
Liquiritin (LQ), the main bioactive constituent of licorice, is a common flavoring and sweetening agent in food products and has a wide range of pharmacological properties, including antidepressant-like, neuroprotective, anti-cancer and anti-inflammatory properties. This study investigated the metabolic pathways of LQ in vitro (rat liver microsomes) and in vivo (rat model) using ultra high-performance liquid chromatography coupled with hybrid triple quadrupole time-of-flight mass spectrometry (UHPLC-QTOF-MS/MS). Moreover, supplementary tools such as key product ions (KPIs) were employed to search for and identify compounds. As a result, 56 in vivo metabolites and 15 in vitro metabolites were structurally characterized. Oxidation, reduction, hydrolysis, methylation, acetylation, and sulfate and glucuronide conjugation were determined to be the major metabolic pathways of $L Q$, and there were differences in LQ metabolism in vitro and in vivo. In addition, the in vitro and in vivo metabolic pathways were compared in this study.
\end{abstract}

\section{Introduction}

Licorice root or Radix glycyrrhizae, a Chinese materia medica (CMM), is widely used to invigorate the spleen, replenish the Qi, dispel heat and remove toxic substances. ${ }^{1}$ In modern medical terms, the biologically active compounds in licorice are triterpene, saponins, flavonoids, polysaccharides and phenolic compounds, ${ }^{2}$ making licorice root a source of medicine and food. These compounds exhibit several important pharmacological activities, including anti-viral, ${ }^{3}$ anti-oxidant, ${ }^{4}$ antibacterial, ${ }^{5}$ anti-inflammatory ${ }^{6}$ and anti-HIV ${ }^{7}$ activities. Moreover, licorice and its extract are widely used in health foods because of the physiological activities of these substances. ${ }^{8}$ Currently, the use of licorice and its extract in grain products, oil products, meat products, beverages, candy, jelly, dried fruit,

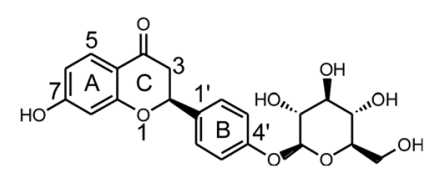

Fig. 1 Chemical structure of $L Q$.

Department of Pharmaceutical Analysis, School of Pharmacy, Hebei Medical University, Shijiazhuang 050017, P. R. China.E-mail: zhanglantong@263.net; Fax: +86-311-86266419; Tel: +86-311-86266419

$\dagger$ Electronic supplementary information (ESI) available: Detailed information regarding other metabolites found in vitro and in vivo. Fig. S1 MS/MS spectra of all metabolites of LQ detected in vitro and in vivo. See DOI: 10.1039/c7ra13760e seeds, soy sauce, etc., are being extensively researched., 90 Among the biologically active compounds in licorice, liquiritin (LQ, Fig. 1) is the dominant component and is considered to be the major active ingredient. ${ }^{11}$

Drug metabolism may lead to detoxification and/or activation reactions, and studies of drug metabolism can aid the identification of active compounds and explain the mechanisms of action of these compounds. It is well known that the liver plays a key role in the metabolism of orally administered drugs..$^{12}$ The rat liver microsome system is often considered as a reasonable model in which to study drug metabolism. On the other hand, in vivo metabolic studies could comprehensively reveal the metabolic pathways of drugs.

In recent years, the use of liquid chromatography coupled with tandem mass spectrometry (LC-MS/MS) has been routinely used to detect and identify metabolites ${ }^{13,14}$ and has been used to study drug metabolism (flavones and flavonoids, ${ }^{15}$ phenylpropanoids, ${ }^{16-18}$ terpenes, ${ }^{19}$ alkaloids, ${ }^{20}$ saponins, ${ }^{21}$ stilbenes ${ }^{22}$ and traditional Chinese medicinal extracts ${ }^{23}$ ), pharmacokinetics and toxicokinetics of metabolites, ${ }^{24,25}$ and tissue distribution and excretion of metabolites. ${ }^{26}$ In addition, this method has applications in lipidomics, ${ }^{27}$ proteomics ${ }^{28}$ and metabolomics. ${ }^{29,30} \mathrm{~A}$ primary advantage of tandem mass spectrometry (MS/MS) is the ability of this method to detect a broad range of drugs with high sensitivity and specificity in a single analytical run. ${ }^{31-34}$ In addition, high-resolution mass spectrometry confirms structures by comparing the exact measured mass of a compound with the exact theoretical mass. ${ }^{35-37}$

To our knowledge, there has been one report of the metabolic profile of LQ China; ${ }^{38}$ however, this report was incomplete 
and only identified 7 metabolites. In this research contribution, a simple and rapid UHPLC-Q-TOF-MS/MS approach combined with pattern recognition analysis was first employed to rapidly screen and characterize metabolites of LQ in vitro and in vivo, which was the first systematic study of the metabolism of LQ in vitro and in vivo. The characterization of 56 in vivo metabolites and 15 in vitro metabolites was achieved by UHPLC-Q-TOF-MS/ MS analysis based on the MS/MS spectra and $\operatorname{clog} P$ values. In addition, the metabolic pathways of LQ were summarized. These results provide insight into the metabolic mechanism of LQ and lay the foundation for novel drug design.

\section{Materials and methods}

\subsection{Chemicals and materials}

LQ (CAS No: 551-15-5) was purchased from Chengdu Desert Biotechnology Co., Ltd. (Chengdu, China). $\beta$-NADP ( $\beta$-nicotinamine adenine dinucleotide phosphate), glucose-6-phosphate (G-6-P) and glucose-6-phosphatedehydrogenase (G-6-PD) were purchased from Sigma Chemical Co. (St. Louis, MO, USA). $\mathrm{MgCl}_{2}$, UDPGA (uridine-5'-diphosphoglucuronic acid trisodium salt), Tris-HCl and alamethicin were purchased from BD Biosciences (Woburn, MA, USA). Phosphate-buffered saline (PBS) was purchased from Sangon Biotech Co., Ltd. (Shanghai, China). HPLC-grade methanol was purchased from J.T. Baker Chemical Company (Phillipsburg, NJ, USA). Formic acid (HPLC grade) was provided by Diamond Technology (Dikma Technologies Inc., Lake Forest, CA, USA). Purified water was obtained from Hangzhou Wahaha Group Co., Ltd. (Hangzhou, China).

\subsection{Instrumentation and conditions}

UHPLC-Q-TOF-MS/MS analysis was performed on a Shimadzu UHPLC system (Shimadzu Corp., Kyoto, Japan) coupled with a triple $\mathrm{TOF}^{\mathrm{TM}} 5600^{+} \mathrm{MS} / \mathrm{MS}$ system (AB Sciex, CA, USA). Information-dependent acquisition (IDA) was carried out. Chromatographic separation was conducted on a Poroshell 120 EC-C $_{18}(2.1 \times 100 \mathrm{~mm}, 2.7 \mu \mathrm{m})$ column with a SecurityGuard $\AA$ UHPLC $\mathrm{C}_{18}$ pre-column (Agilent Corp, Santa Clara, CA, USA). The column temperature was maintained at $25^{\circ} \mathrm{C}$. The mobile phase was consisted of water containing $0.1 \%$ formic acid (A) and methanol (B). The gradient elution program was optimized for the separation, and the program was as follows: 0-2 min, 1015\% B; 2-15 min, 15-45\% B; 15-20 min, 45-95\% B; 20-25 min, $95-95 \%$ B. After maintaining the column at $95 \%$ solvent B for $5 \mathrm{~min}$, the column was returned to its starting conditions over $1 \mathrm{~min}$ and was equilibrated in $10 \%$ solvent $\mathrm{B}$ for $5 \mathrm{~min}$. The flow rate of the mobile phase was set to $0.3 \mathrm{~mL} \min ^{-1}$, and the injection volume was $3 \mu \mathrm{L}$.

A Triple TOF $^{\mathrm{TM}} 5600$ system with DuoSpray ${ }^{\mathrm{TM}}$ ion sources (AB Sciex Triple TOF ${ }^{\mathrm{TM}} 5600+$, Concord, Ontario, Canada) operating in the negative electrospray ionization mode was used for detection. The following MS/MS conditions were used: ion spray voltage, $-4.5 \mathrm{kV}$; the turbo spray temperature, $550{ }^{\circ} \mathrm{C}$; and declustering potential (DP), $-60 \mathrm{~V}$. Nitrogen was used as the nebulizer and auxiliary gas. Furthermore, the flows of the nebulizer gas (gas 1), heater gas (gas 2) and curtain gas were set to 55,55 and $35 \mathrm{~L} \mathrm{~min}^{-1}$, respectively. The collision energy (CE) was set to $-35 \mathrm{eV}$, and the collision energy spread (CES) was $15 \mathrm{eV}$.

Metabolite identification was performed with MetabolitePilot 1.5 (AB Sciex, CA, USA) based on accurate measurements of $m / z$ values and on the processing of the data obtained from the XIC (extracted ion chromatography), MDF (mass defect filtering), PIF (product ion filtering) and NLF (neutral loss filtering) screening of putative metabolites. In addition, elemental compositions and chemical formulas were calculated.

\subsection{Animals and drug administration}

Thirty male Sprague-Dawley (SD) rats, 10-12 weeks in age and weighing 200-230 g, were obtained from the Experimental Animal Center of Hebei (Shijiazhuang, China). Rats were housed under standard temperature, humidity and light conditions. The animals were kept in an environmentally controlled breeding room for 7 days and fasted for $12 \mathrm{~h}$ before experiments. LQ was dissolved in a $0.5 \%$ carboxymethyl cellulose sodium (CMC-Na) aqueous solution. Thirty male SD rats were divided into six groups of five rats per group, which included experimental blood, urine and feces, and bile groups as well as blank blood, urine and feces, and bile groups. The prepared LQ suspension was orally administered to 15 rats from the experimental blood, urine and feces, and bile groups at a dose of $120 \mathrm{mg} \mathrm{kg}^{-1}$, and $0.5 \%$ CMC-Na aqueous solution was orally administered to 15 rats from the blank blood, urine and feces, and bile groups. All experiments were conducted in accordance with the guides of Animal Care and Use Committee at Hebei Medical University. This study was also performed in strict accordance with the NIH guidelines for the care and use of laboratory animals (NIH Publication No. 85-23 Rev. 1985) and was approved by the Institutional Animal Care and Use Committee of National Tissue Engineering Center (Shanghai, China).

Plasma sample (five SD rats) collection was performed as follows: blood was taken from the canthi of the rats $0.17,0.50$, $0.75,1,2,4,6,9,12$ and $24 \mathrm{~h}$ after administration. After centrifugation at $1400 \times g$ for 5 min (Hunan Xiangyi Laboratory Instrument Development Co. Ltd., Hunan, China), the supernatant was collected, and all plasma samples were combined. Blank plasma was collected in the same manner from rats (five) administered 0.5\% CMC-Na aqueous solution.

Urine and feces (five SD rats) collection was performed as follows: urine and feces were collected during the $0-4 \mathrm{~h}, 4-8 \mathrm{~h}$, 8-12 h, 12-24 h, 24-36 h, 36-48 h, 48-60 h and 60-72 h periods after administration, and all the urine and feces samples were combined. Rats (five) administered 0.5\% CMC-Na aqueous solution were subjected to the same process to collect blank urine and feces samples.

Bile (five SD rats) collection was performed as follows: rats were administered urethane-containing physiological saline solution (1.5-2 $\mathrm{g} \mathrm{kg}^{-1}$ ) after gavage, and then, bile duct cannulation. Then, bile samples were collected during $0-1 \mathrm{~h}, 1-3 \mathrm{~h}$, 3-5 h, 5-8 h, 8-12 h, 12-20 h and 20-24 h periods after 
administration. Finally, all bile samples were consolidated. Rats (five) administered 0.5\% CMC-Na aqueous solution were subjected to the same process to collect a blank bile sample.

Three milliliters of blood, urine and bile samples were taken, and the protein in the samples was precipitated by methanol. Then, the supernatant was concentrated to dryness under reduced pressure at $25{ }^{\circ} \mathrm{C}$ using a Heidolph Laborota 4001 rotatory evaporator (Heidolph Instruments, $\mathrm{GmbH} \& \mathrm{Co}$, Schwabach, Germany). The dried samples were dissolved in 300 $\mu \mathrm{L}$ of methanol in an ultrasonic bath for $5 \mathrm{~min}$, and then, the samples were centrifuged for $10 \mathrm{~min}$ at $10000 \times \mathrm{g}$. Then, the supernatant was injected into the UHPLC-Q-TOF-MS/MS system for further analysis.

Methanol $(20 \mathrm{~mL})$ was added to the feces sample $(2.0 \mathrm{~g})$, and then, the sample was ultrasonicated for 45 min (Kun Shan Ultrasonic Instruments Co., Kunshan, China). After the mixture was centrifuged at $10000 \times g$ for $10 \mathrm{~min}$, the supernatant was collected and blow-dried in a nitrogen atmosphere. The residue was dissolved in $400 \mu \mathrm{L}$ of methanol and centrifuged at 10000 $\times g$ for $10 \mathrm{~min}$. The supernatant $(3 \mu \mathrm{L})$ was injected into the chromatographic instrument.

All the bio-samples were placed in the $-80{ }^{\circ} \mathrm{C}$ freezer for storage.

\subsection{Microsomal incubation}

2.4.1. Preparation of standard solutions. The appropriate amount of standard LQ was accurately weighed and dissolved in $50 \%$ methanol to make the standard solution $\left(2.15 \mathrm{mg} \mathrm{mL}^{-1}\right)$. The solution was stored at $4{ }^{\circ} \mathrm{C}$ in a refrigerator.

2.4.2. Preparation of liver microsomes. Liver microsomes were prepared by differential centrifugation. ${ }^{39}$ All surgical instruments and experimental reagents were stored at $4{ }^{\circ} \mathrm{C}$ in refrigerator. Five male SD rats (220-250 g) were fasted for $24 \mathrm{~h}$ and decapitated. The liver was quickly retrieved, and the blood was blotted with filter paper. Then, the liver was weighed and was repeatedly washed with sugar solution till a khaki color. Then, the liver was added to an ice-cold sugar solution that was 4 times the weight of the liver and was then cut and homogenized. After centrifugation at $20000 \times g$ for $20 \mathrm{~min}$ at $4{ }^{\circ} \mathrm{C}$, the precipitate was discarded. After additional centrifugation at $100000 \times g$ for $60 \mathrm{~min}$ at $4{ }^{\circ} \mathrm{C}$, the supernatant was discarded. The precipitate was washed with 4 times as much cold Tris- $\mathrm{HCl}$ solution. After centrifugation at $100000 \times g$ for $60 \mathrm{~min}$ at $4{ }^{\circ} \mathrm{C}$, the precipitation obtained was resuspended using 4 times as much Tris-HCl solution to obtain liver microsomes. Finally, the liver microsomes were placed at $-80{ }^{\circ} \mathrm{C}$ in the freezer for storage until further use. In addition, the protein concentration of the liver microsome suspension was determined by the Lowry method. ${ }^{40}$

2.4.3. Phase I metabolism. The typical incubation mixture (200 $\mu \mathrm{L}$ final volume) consisted of a $0.1 \mathrm{~mol} \mathrm{~L}^{-1} \mathrm{~K}_{2} \mathrm{HPO}_{4}$ buffer containing rat microsomes $\left(1.0 \mathrm{mg} \mathrm{mL}{ }^{-1}\right), 3.3 \mathrm{mmol} \mathrm{L}{ }^{-1} \mathrm{MgCl}_{2}$, $1.3 \mathrm{mmol} \mathrm{L}^{-1} \beta$-NADPH, $3.3 \mathrm{mmol} \mathrm{L}^{-1}$ glucose-6-phosphate, 1.0 $\mathrm{U} \mathrm{mL}^{-1}$ glucose-6-phosphate dehydrogenase and $100 \mu \mathrm{mol} \mathrm{L}^{-1}$ LQ methanol (final amount in the reaction medium: 1\%). Preincubation was carried out at $37{ }^{\circ} \mathrm{C}$ for $5 \mathrm{~min}$, and then,
NADPH was added to initiate the reaction. After incubation for $30 \mathrm{~min}$ at $37^{\circ} \mathrm{C}$ in a metabolic shaker $(1200 \times g$, Hangzhou Miu Instruments Co., Ltd., Hangzhou, China), $1 \mathrm{~mL}$ of ice-cold ethyl acetate was added to stop the reaction, and the mixture was vortexed for $5 \mathrm{~min}$. After centrifugation at $10000 \times g$ for $10 \mathrm{~min}$, the organic phase was collected and evaporated under nitrogen gas. Residues were redissolved in $100 \mu \mathrm{L}$ of methanol, and an aliquot $(3 \mu \mathrm{L})$ was injected into the chromatographic system for analysis. The blank sample was incubated without LQ, while the control sample was incubated without the NADPH-generating system by following the method described above.

2.4.4. Phase II metabolism. The reaction mixture (total volume $200 \mu \mathrm{L}$ ) containing $1.0 \mathrm{mg} \mathrm{mL}^{-1}$ rat liver microsomes, $2 \mathrm{mmol} \mathrm{L}{ }^{-1}$ UDPGA, $8 \mathrm{mmol} \mathrm{L}{ }^{-1} \mathrm{MgCl}_{2}, 25 \mu \mathrm{g} \mathrm{mL}{ }^{-1}$ alamethicin in PBS (pH 7.4) and $100 \mu \mathrm{mol} \mathrm{L}^{-1} \mathrm{LQ}$ methanol (final amount in the reaction medium: 1\%) solution was preincubated for $20 \mathrm{~min}$ at $37^{\circ} \mathrm{C}$, and then, UDPGA was added to start the reaction. After incubation for $60 \mathrm{~min}$ at $37^{\circ} \mathrm{C}$ in a metabolic shaker $(1200 \times g), 200 \mu \mathrm{L}$ of ice-cold methanol was added to stop the reaction. After vortexing for $5 \mathrm{~min}$, the organic phase was collected and evaporated under nitrogen gas. Methanol $(100 \mu \mathrm{L})$ was added to the residues, and an aliquot $(3 \mu \mathrm{L})$ was injected into the chromatographic system for analysis. The blank sample was incubated without LQ, while the control sample was incubated without the UDPGA-generating system following the method described above.

\section{Results and discussion}

A total of 56 in vivo metabolites and 15 in vitro metabolites were detected in the experimental conditions used. The metabolites detected in vivo and in vitro are listed in Tables 1 and 2, respectively, and the structures of the metabolites are shown in Fig. 2. The XIC and MS/MS data of the metabolites detected in vivo and in vitro are presented in Fig. 3 and S1, $\dagger$ respectively.

\subsection{Analytical strategy and metabolite analysis}

In this study, a method of metabolite identification was developed that was based on a Triple TOF ${ }^{\mathrm{TM}}$ instrument with a multiple mass defect filter (MMDF) combined with dynamic background subtraction (DBS)-dependent on-line data acquisition and multiple post-acquisition data mining technologies. First, on-line data and accurate MS/MS data were acquired using a full-scan, unique and effective MMDF and a DBSdependent data acquisition method. ${ }^{41}$ Then, post-acquisition data mining was performed using various data-mining tools such as XIC, MDF, PIF and NLF. ${ }^{42}$ Furthermore, structures of the metabolites of LQ were elucidated based on accurate mass measurements, knowledge of relevant drug bio-transformation, previously investigated fragmentation patterns of LQ, and MS/ MS spectra of metabolites. Peakview 1.2 software was used to identify possible metabolites by comparing the extracted ion chromatograms and base peak chromatograms of the sample group with those of the blank group. ${ }^{43}$ Finally, the useful clog $P$ parameter, which was calculated using the ChemDraw Ultra 12.0 program, was introduced to distinguish between structural 


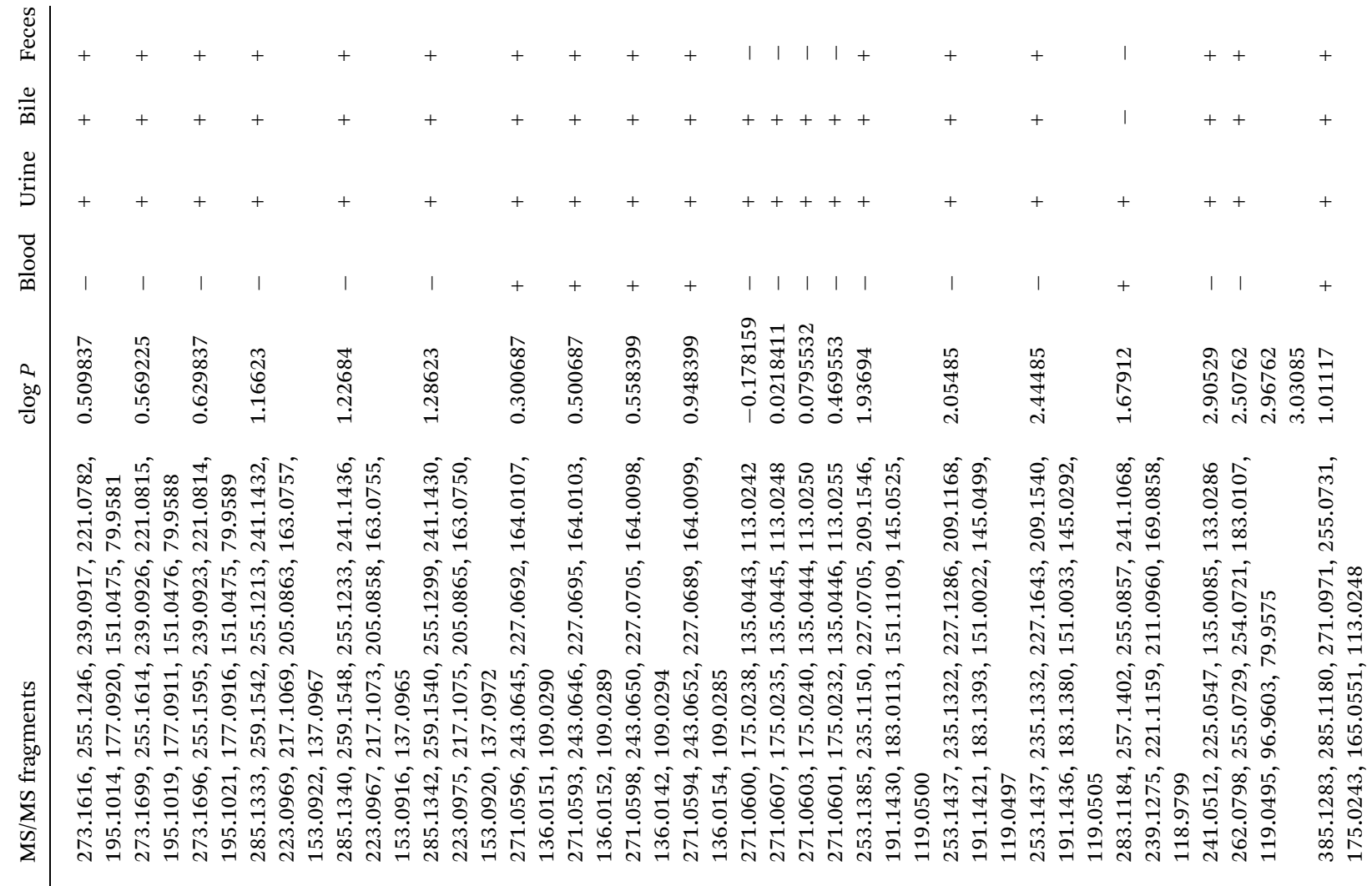

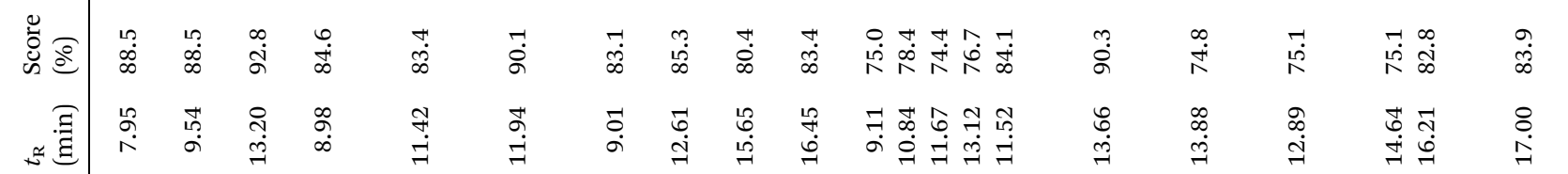

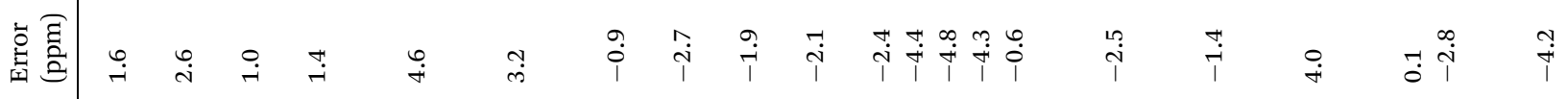

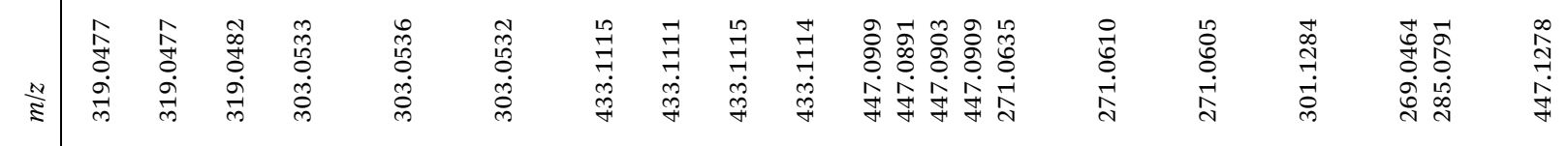

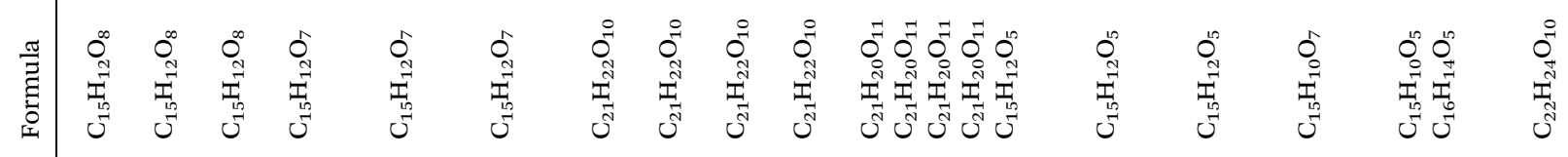

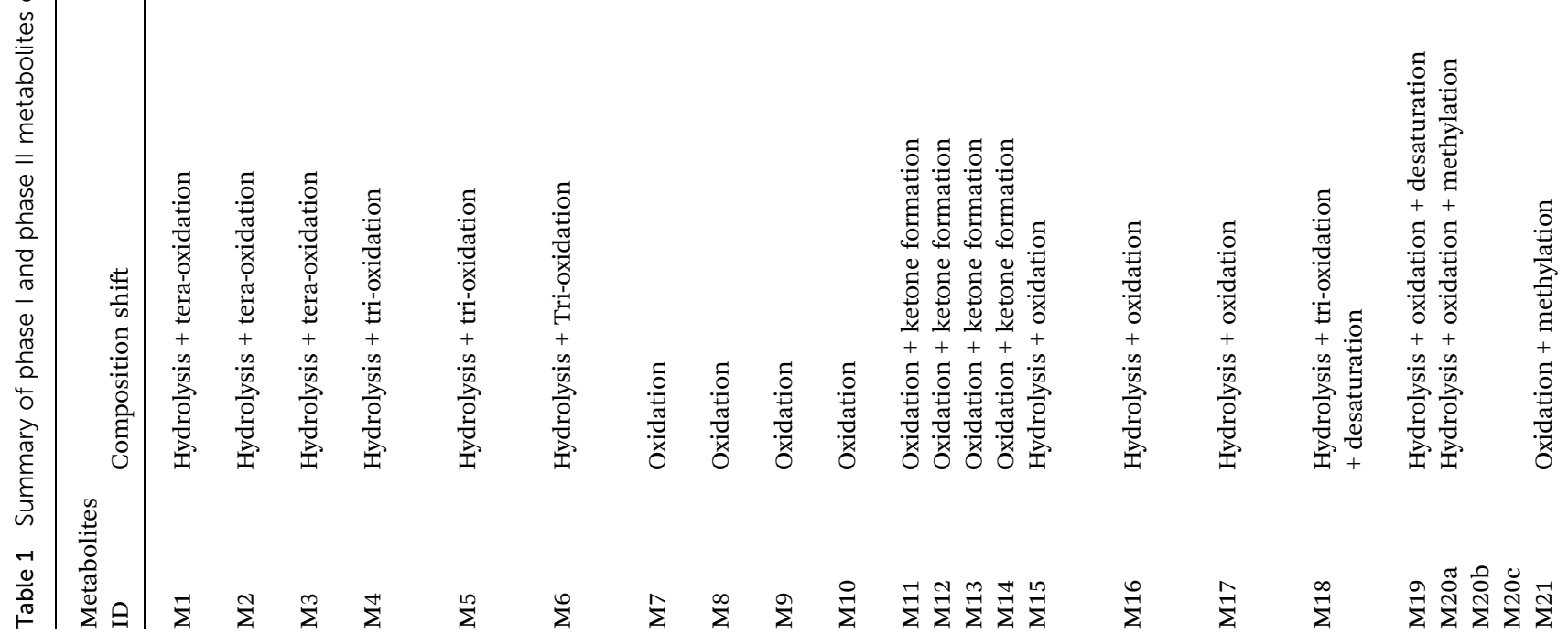




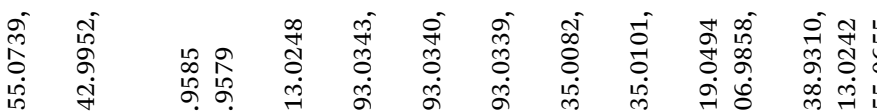

ป

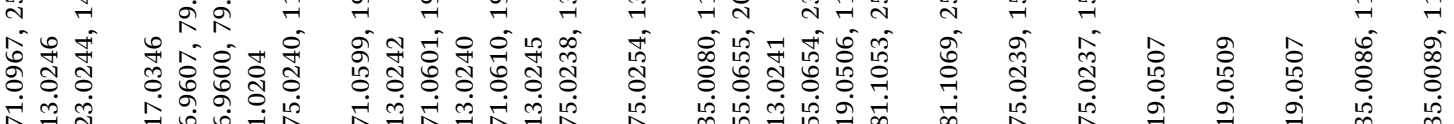

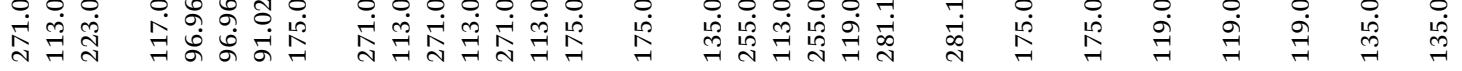

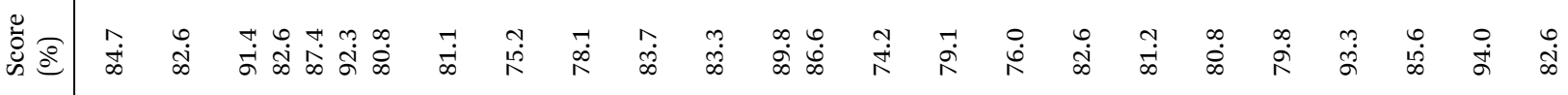

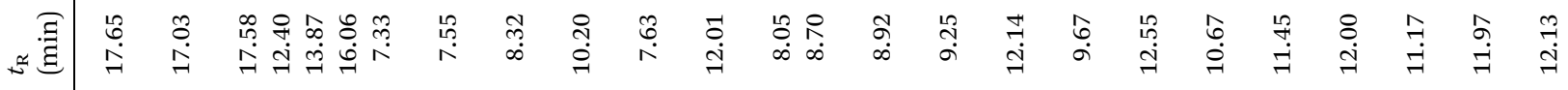

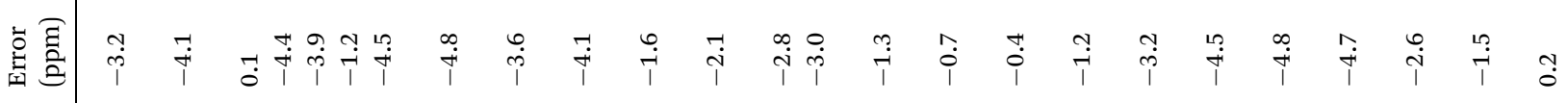

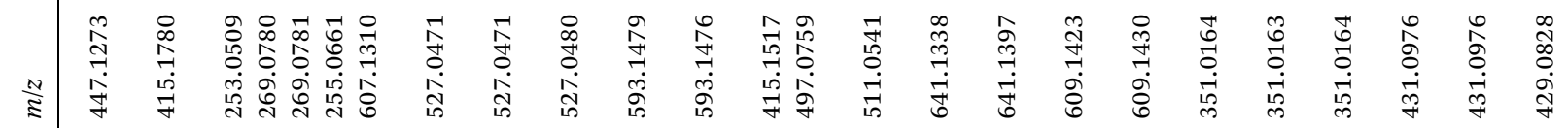

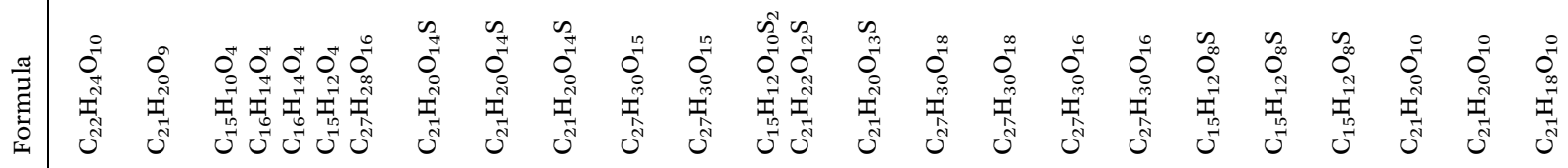

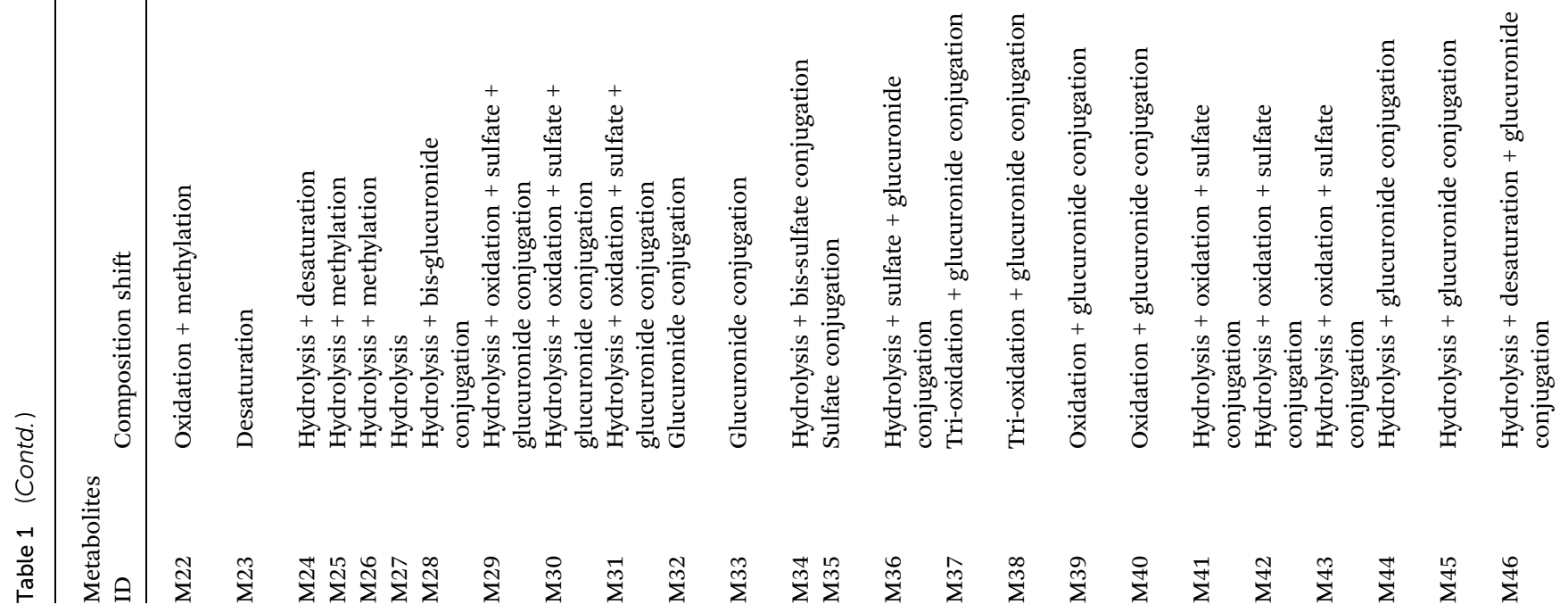




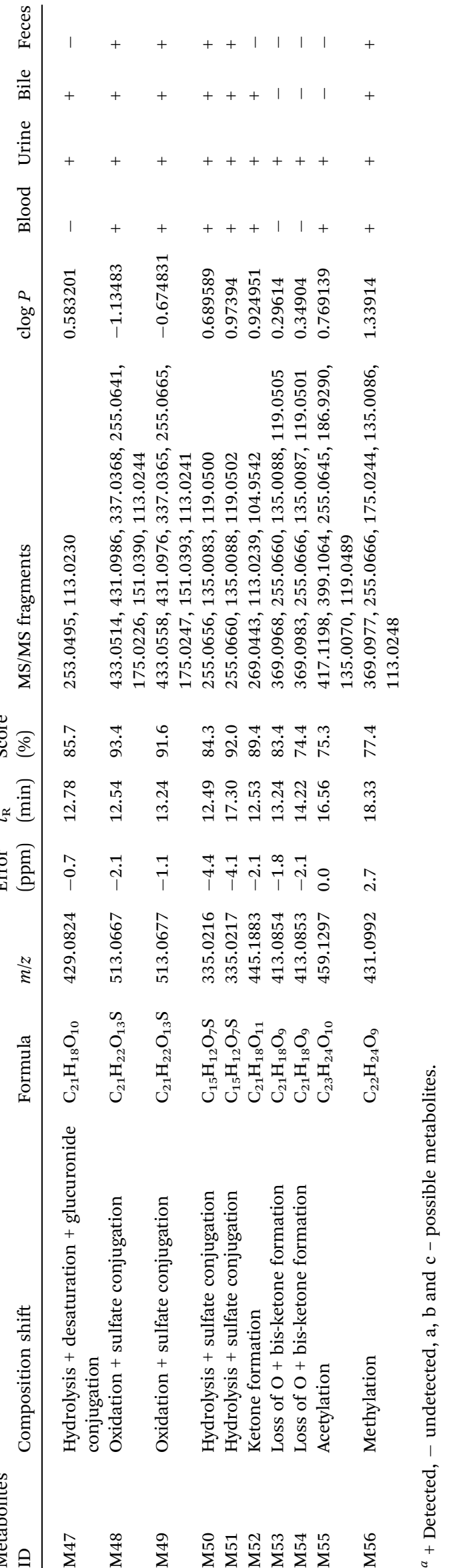

isomers. Generally, compounds with the larger values have longer retention times in reversed-phase liquid chromatography systems. ${ }^{\mathbf{4 4 , 4 5}}$

All chemical constituents in TMM can be categorized into different families based on structural types. Thus, groups of compounds with identical carbon skeletons may yield similar fragmentation patterns and then generate the same characteristic fragment ions when subjected to collision-induced dissociation (CID) for mass spectrometry. Accordingly, a core supplementary tool in this approach is to use key product ions (KPIs) as markers for compounds detection and identification. ${ }^{46-48}$ In this study, a KPI at $m / z 255.0651$ could be generated from common substructures as standards and was selected as a diagnostic ion for detecting relevant analogues of these substructures (shown in Fig. 3).

\subsection{Mass fragmentation behavior of LQ}

The retention time of LQ was $12.01 \mathrm{~min}$, and LQ produced a molecular ion $[\mathrm{M}-\mathrm{H}]^{-}$at $m / z 417.1176$ under the experimental conditions. Moreover, major secondary fragment ions at $m / z 255.0651\left[\mathrm{M}-\mathrm{H}-\mathrm{C}_{6} \mathrm{H}_{10} \mathrm{O}_{5}\right]^{-}, 135.0079$ (RDA reaction (retro Diels-Alder reaction)), 119.0496 (RDA reaction) and 91.0190 (RDA reaction) were detected. The proposed MS/MS fragmentation behavior and fragmentation pathways of LQ are shown in Fig. 4. The molecular weight and elemental composition of LQ were used as a baseline for comparison with some metabolites.

\subsection{Identification of in vivo phase I metabolites}

\subsubsection{Oxidation reaction}

Metabolites M1, M2 and M3. M1-M3 were eluted at 7.95, 9.54 and $13.20 \mathrm{~min}$, respectively, with deprotonated molecular ions $[\mathrm{M}-\mathrm{H}]^{-}$at $m / z 319.0477,319.0477$ and 319.0482, respectively, which were 98 Da less than the value obtained for LQ and corresponded to $\mathrm{C}_{15} \mathrm{H}_{12} \mathrm{O}_{8}$. The fragment ions at $\mathrm{m} / \mathrm{z} 273.1696$, 255.1595, 239.0923, 221.0814 and 151.0475 were produced from $\mathrm{M} 1, \mathrm{M} 2$ and $\mathrm{M} 3$ by the loss of $\mathrm{CO}, \mathrm{H}_{2} \mathrm{O}, \mathrm{O}$ and by RDA reaction. Among these fragments, the fragment ion at $m / z 255.1595$ was hydrolyzed LQ. M1, M2 and M3 were oxidation metabolites of LQ and were assigned based on $\operatorname{clog} P$ values of 0.509837 , 0.569225 and 0.629837 by ChemDraw 12.0 software.

Metabolites M7, M8, M9 and M10. M7-M10, eluted at 9.01, 12.61, 15.65 and $16.45 \mathrm{~min}$, respectively, were characterized as deprotonated molecular ions $[\mathrm{M}-\mathrm{H}]^{-}$at $\mathrm{m} / \mathrm{z}$ 433.1115, 433.1111, 433.1115 and 433.1114, respectively, which were 16 Da more than the value obtained for the parent drug LQ. This finding suggested that M7-M10 were oxidation metabolites of LQ and that the chemical formula of M7-M10 was $\mathrm{C}_{21} \mathrm{H}_{22} \mathrm{O}_{10}$. The observed diagnostic fragment ions at $\mathrm{m} / \mathrm{z}$ 271.0596, 243.0645, 227.0692, 136.0151 and 109.0290 were generated by the loss of $\mathrm{C}_{6} \mathrm{H}_{10} \mathrm{O}_{5}, \mathrm{CO}$ and $\mathrm{O}$ and by RDA reaction. The clog $P$ values of $\mathrm{M} 7-\mathrm{M} 10$ were $0.300687,0.500687,0.558399$ and 0.948399 , respectively. Therefore, the four compounds were immediately identified on the basis of the retention times and clog $P$ values.

Metabolites M11, M12, M13 and M14. M11-M14 were eluted at 9.11, 10.84, 11.67 and $13.12 \mathrm{~min}$, respectively, with 
Table 2 Summary of phase I and phase II metabolites of LQ in vitro ${ }^{a}$

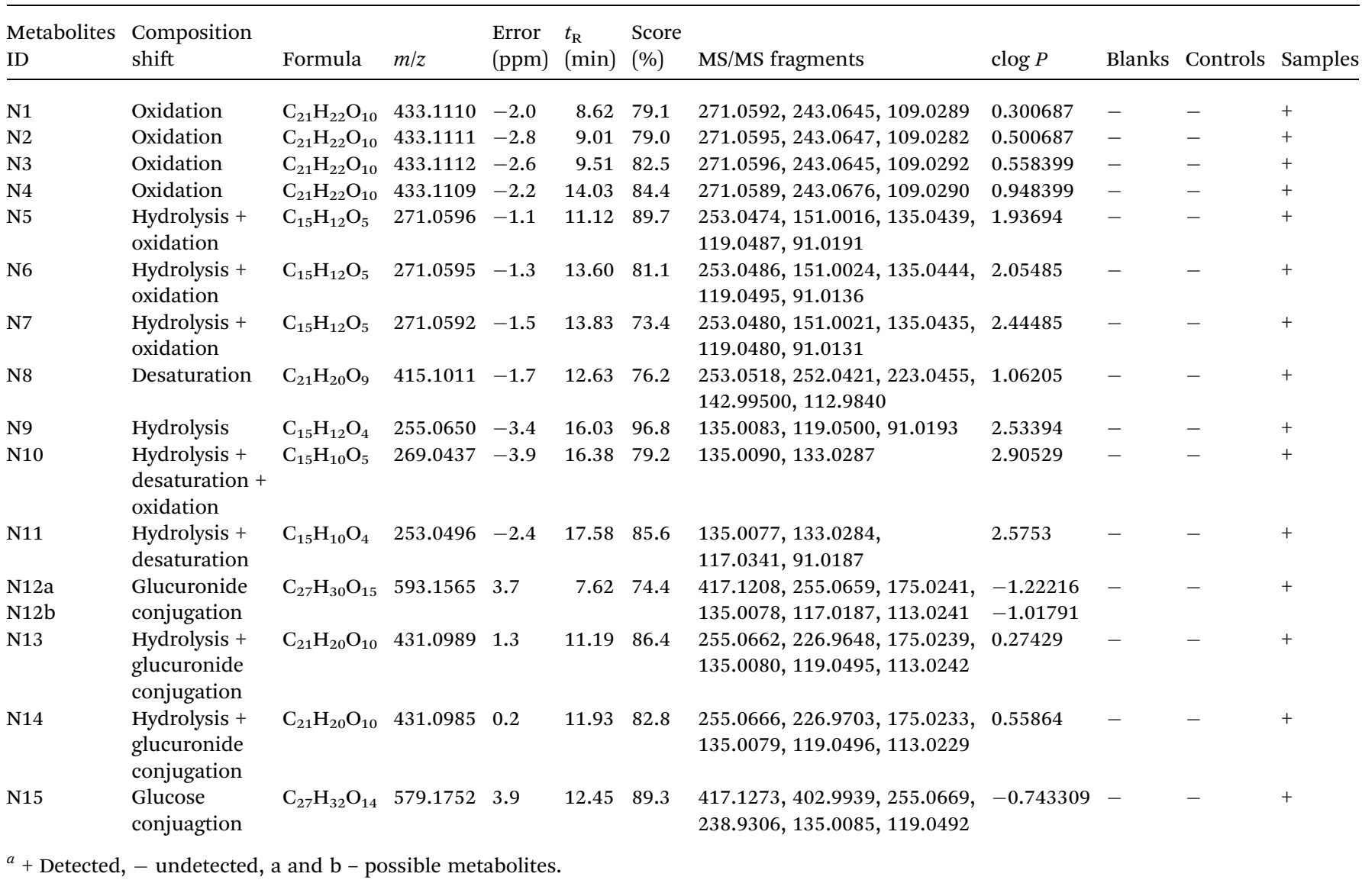

deprotonated molecular ions $[\mathrm{M}-\mathrm{H}]^{-}$at $\mathrm{m} / \mathrm{z}$ 447.0909, 447.0891, 447.0903 and 447.0909, respectively, which were 30 Da more than the value obtained for LQ. The major fragment ions detected at $\mathrm{m} / \mathrm{z} 271.0600,135.0443$ and 113.0242 were generated by the loss of $\mathrm{C}_{6} \mathrm{H}_{8} \mathrm{O}_{6}$ and by RDA reaction, which implied that M11-M14 were oxidation products of LQ and that the chemical formula was $\mathrm{C}_{21} \mathrm{H}_{20} \mathrm{O}_{11}$. In addition, M11, M12, M13 and M14 were assigned based on the $\operatorname{cog} P$ values of M11, M12, M13 and M14, which were $-0.178159,0.0218411$, 0.0795532 and 0.469553 , respectively.

Metabolite M18. M18 was detected at a retention time of $12.89 \mathrm{~min}$, with a deprotonated molecular ion $[\mathrm{M}-\mathrm{H}]^{-}$at $\mathrm{m} / \mathrm{z}$ 301.1284, 46 Da higher than that of M0 losing $\mathrm{C}_{6} \mathrm{H}_{10} \mathrm{O}_{5}$, which suggested that M18 was oxidation metabolite. The fragment ions at $\mathrm{m} / \mathrm{z} 283.1184,255.0857,239.1275,169.0858$ and 118.9799 were gained by loss of $\mathrm{H}_{2} \mathrm{O}, \mathrm{CO}, \mathrm{O}$ and by RDA reaction. According to the fragmentation, the chemical formula of M18 was $\mathrm{C}_{15} \mathrm{H}_{10} \mathrm{O}_{7}$.

Metabolite M19. M19 was detected at a retention time of 14.64 min with a deprotonated molecular ion $[\mathrm{M}-\mathrm{H}]^{-}$at $\mathrm{m} / \mathrm{z}$ 269.0464, which was generated by the loss of two oxygens from M18. Typical fragment ions at $m / z$ 241.0512, 225.0547, 135.0085 and 133.0286 were detected due to successive loss of $\mathrm{CO}$ and $\mathrm{O}$ and due to RDA reaction. Thus, M19 was deduced to be $\mathrm{C}_{15} \mathrm{H}_{10} \mathrm{O}_{5}$.
Metabolite M20. M20 had a retention time of $16.21 \mathrm{~min}$ and was detected at $m / z 285.0791\left([\mathrm{M}-\mathrm{H}]^{-}\right)$, which was 30 Da more than the value obtained for hydrolyzed LQ, which implied that oxidation and methylation reactions had occurred. Representative fragment ions were observed at $\mathrm{m} / \mathrm{z} 255.0729$ and 119.0495 due to loss of $\mathrm{CH}_{2} \mathrm{O}$ and due to RDA reaction, which suggested that the formula of $\mathrm{M} 20$ was $\mathrm{C}_{16} \mathrm{H}_{14} \mathrm{O}_{5}$.

Metabolite M23. A peak was eluted at a retention time of $17.03 \mathrm{~min}$. The MS/MS spectrum of M23 showed a deprotonated molecular ion $[\mathrm{M}-\mathrm{H}]^{-}$at $m / z$ 415.1780, which was 2 Da less than the value obtained for $\mathrm{M} 0$, which confirmed the molecular formula to be $\mathrm{C}_{21} \mathrm{H}_{20} \mathrm{O}_{9}$. In addition, the MS/MS spectrum of M23 showed a number of characteristic fragment ions at $\mathrm{m} / \mathrm{z}$

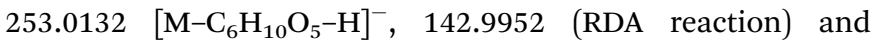
112.9901 (RDA reaction).

\subsubsection{Reduction reaction}

Metabolite M24. M24 was detected at $17.58 \mathrm{~min}$ and showed a deprotonated molecular ion $[\mathrm{M}-\mathrm{H}]^{-}$at $m / z$ 253.0509, which was 164 Da lower than the value obtained for M0, which suggested that the loss of two hydrogens from hydrolyzed M0. Meanwhile, representative fragment ions at $\mathrm{m} / \mathrm{z}$ 135.0082, 133.0291 and 117.0346 were obtained due to RDA reaction. The formula was identified as $\mathrm{C}_{15} \mathrm{H}_{10} \mathrm{O}_{4} \cdot{ }^{38}$

\subsubsection{Hydrolysis reaction}

Metabolites M25, M26. M25 and M26 displayed molecular ions $[\mathrm{M}-\mathrm{H}]^{-}$at $m / z 269.0780$ and 269.0781, respectively, and 


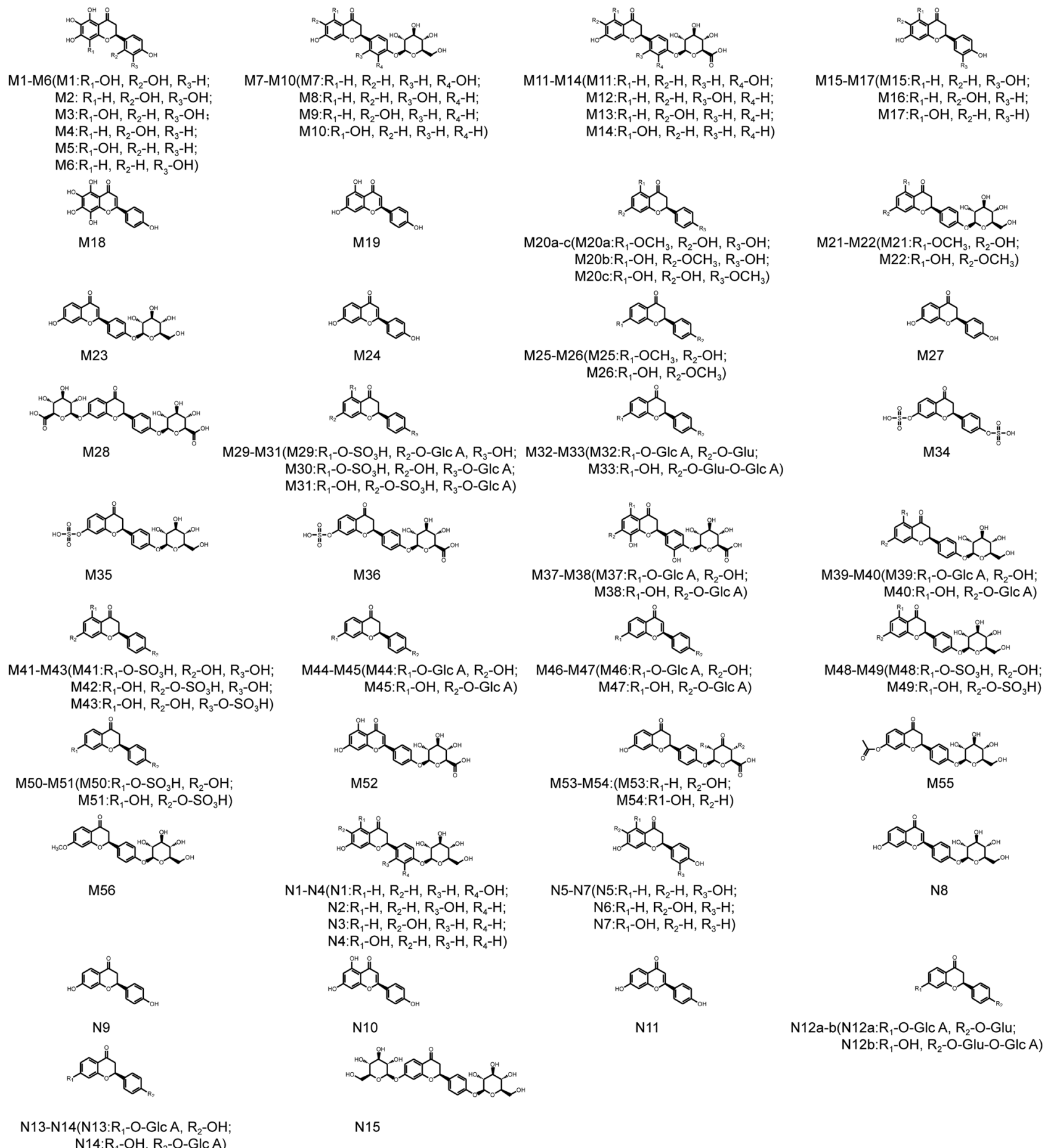

Fig. 2 Chemical structures of all metabolites of $L Q$ detected in vitro and in vivo ( $a, b$ and c-possible chemical structure).

had retention times of 12.40 and $13.87 \mathrm{~min}$, respectively. The masses of the deprotonated M25 and M26 were 14 Da higher than the mass of hydrolyzed LQ, which suggested that a methylation reaction occurred on LQ after the loss of $\mathrm{C}_{6} \mathrm{H}_{10} \mathrm{O}_{5}$. The MS/MS spectra showed a characteristic fragment ion at $\mathrm{m} / \mathrm{z}$ 253.0637 $[\mathrm{M}-\mathrm{O}-\mathrm{H}]^{-}$, which implied that the formula of M25 and M26 was $\mathrm{C}_{16} \mathrm{H}_{14} \mathrm{O}_{4}$. Then, M25 and M26 were identified based on their $\operatorname{cog} P$ values of 2.83559 and 3.11994, respectively.
Metabolite M27. The metabolite M27 exhibited a deprotonated ion $[\mathrm{M}-\mathrm{H}]^{-}$at $m / z$ 255.0661, which was 162 Da lower than the value obtained for M0, confirming the occurrence of hydrolysis. In addition, typical fragment ions at $\mathrm{m} / \mathrm{z} 135.0090$, 119.0510 and 91.0204 were produced by $\mathrm{RDA}$ reaction. The formula of $\mathrm{M} 27$ was deduced to be $\mathrm{C}_{15} \mathrm{H}_{12} \mathrm{O}_{4} \cdot{ }^{38}$ 

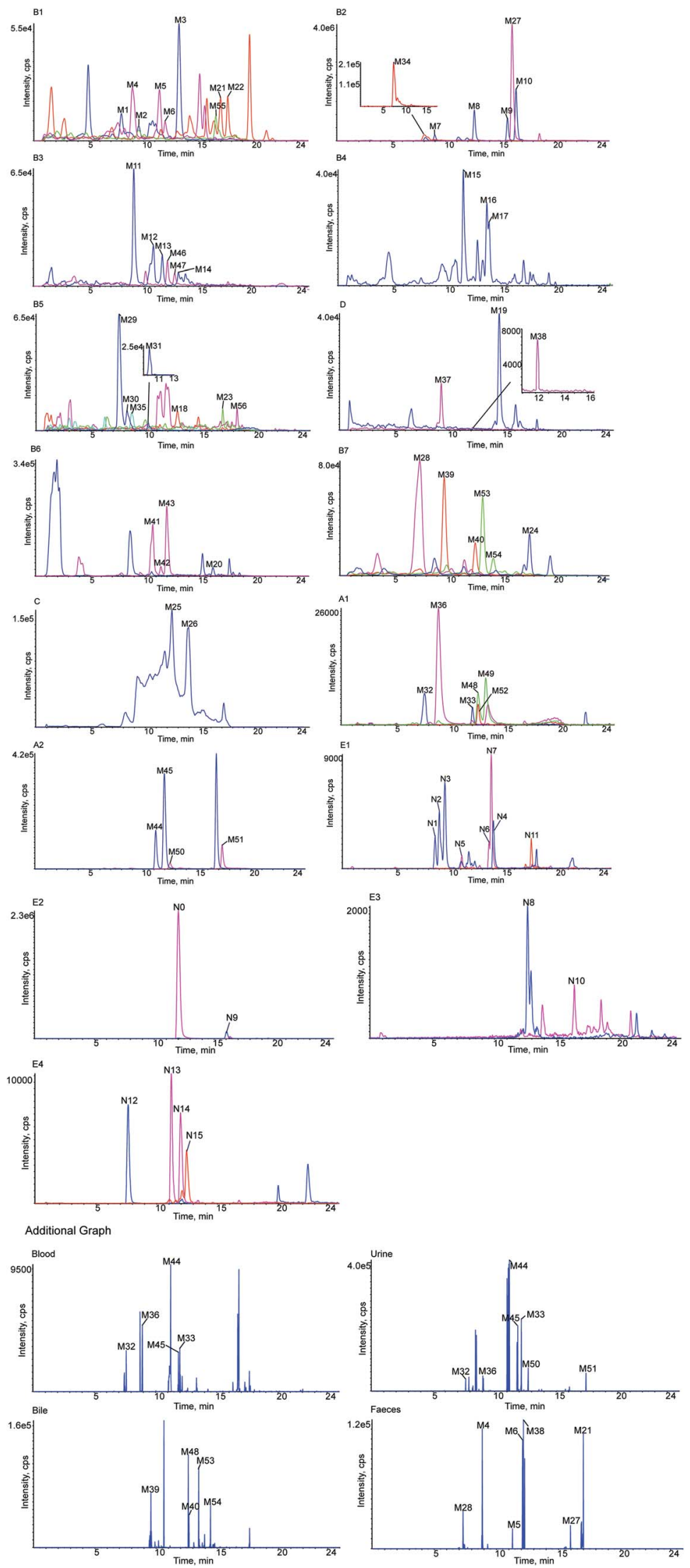

Fig. 3 Extracted ion chromatograms of all metabolites of $L Q$ detected in vitro and in vivo. (A1-2 in rat blood sample, B1-7 in rat urine sample, $\mathrm{C}$ in rat bile sample, D in rat feces sample, E1-4 in rat liver microsomes). Additional graph: metabolites detected by KPIs in rat blood, urine, bile and feces samples. 

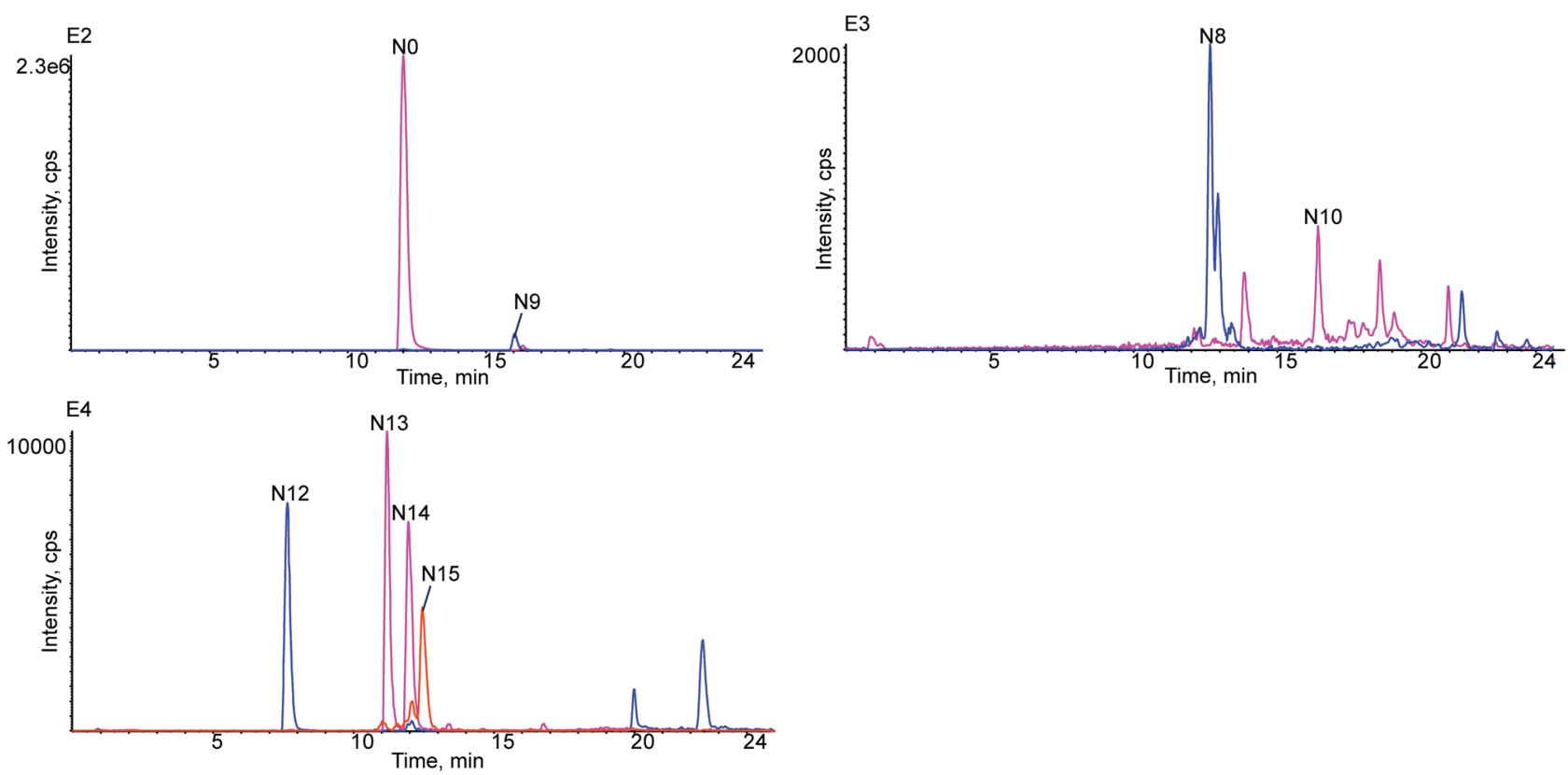

Additional Graph
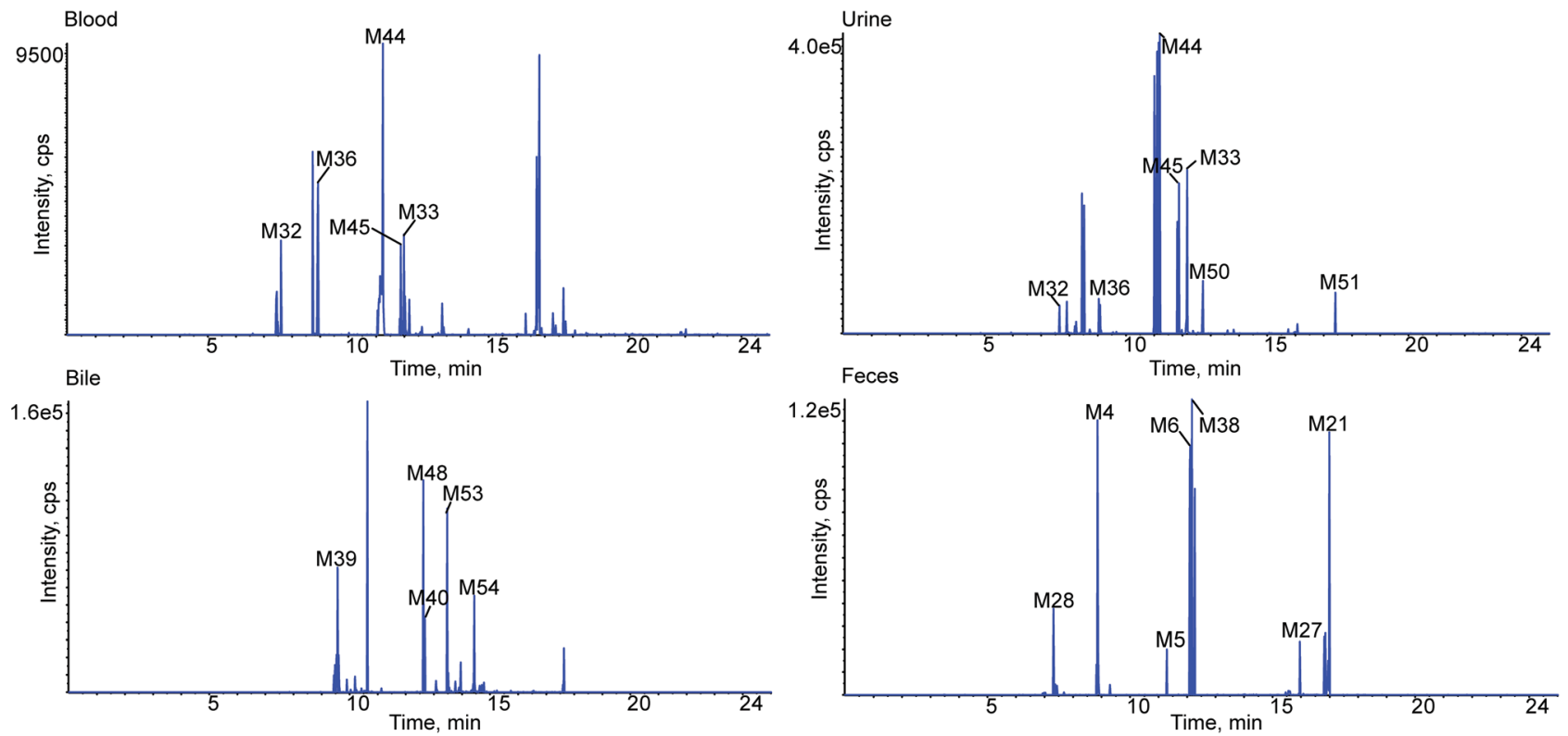

Fig. 3 (Contd.)

\subsection{Identification of in vivo phase II metabolites}

3.4.1. Metabolite M28. A peak was detected at $7.33 \mathrm{~min}$ and exhibited a deprotonated molecular ion $[\mathbf{M}-\mathrm{H}]^{-}$at $\mathrm{m} / \mathrm{z}$ 607.1310, which was $190 \mathrm{Da}$ higher than the value obtained for LQ. Secondary fragment ions were detected at $\mathrm{m} / \mathrm{z} 431.0975$ [M$\left.\mathrm{C}_{6} \mathrm{H}_{8} \mathrm{O}_{6}-\mathrm{H}\right]^{-}$and $255.0655\left[\mathrm{M}-2 \mathrm{C}_{6} \mathrm{H}_{8} \mathrm{O}_{6}-\mathrm{H}\right]^{-}$, suggesting that hydrolyzed LQ had undergone bis-glucuronide conjugation. In addition, the chemical formula was deduced to be $\mathrm{C}_{27} \mathrm{H}_{28} \mathrm{O}_{16}$.

3.4.2. Metabolites M29, M30, M31. M29-M31 were identified as sulfate and glucuronide conjugation metabolites. M29M31 were eluted at 7.55, 8.32 and $10.20 \mathrm{~min}$ and had deprotonated molecular ions $[\mathrm{M}-\mathrm{H}]^{-}$at $m / z 527.0471,527.0471$ and 527.0480 , which were 110 Da more than the value obtained for
M0. The product ions detected at $\mathrm{m} / \mathrm{z} 447.0917,271.0599$, 175.0237, 135.0081 and 113.0242 were produced by the loss of $\mathrm{SO}_{3}$ and $\mathrm{C}_{6} \mathrm{H}_{8} \mathrm{O}_{6}$ and by RDA reaction, which suggested that the formula was $\mathrm{C}_{21} \mathrm{H}_{20} \mathrm{O}_{14} \mathrm{~S}$. In addition, the clog $P$ values of M29, M30 and M31 were -1.91668, -1.61368 and -1.15368, respectively, and M29, M30 and M31 were identified based on this information.

3.4.3. Metabolites M32, M33. Two peaks were eluted at 7.63 and 12.01 min under the chromatography conditions used. M32 and M33 were isomers and had identical MS/MS spectra. M32 and M33 had deprotonated molecular ions $[\mathrm{M}-\mathrm{H}]^{-}$at $\mathrm{m} / \mathrm{z}$ 593.1479 and 593.1476, respectively, which were $176 \mathrm{Da}$ higher than the value obtained for M0, suggesting that M32 and M33 were glucuronide conjugation metabolites. Moreover, the MS/ 

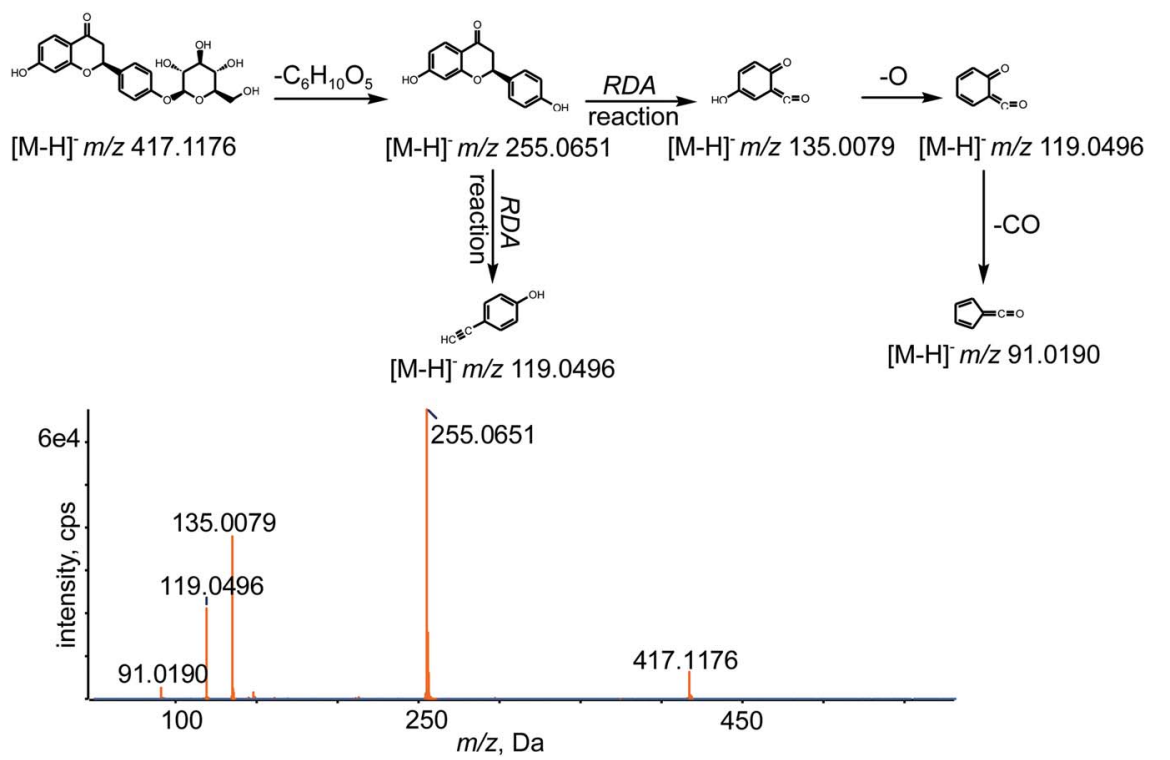

Fig. $4 \mathrm{MS} / \mathrm{MS}$ spectrum of $L Q$ and its predominant fragmentation pathways.

MS spectra showed fragment ions at $m / z$ 417.1143, 255.0658, 135.0101 and 119.0517 due to the loss of $\mathrm{C}_{6} \mathrm{H}_{8} \mathrm{O}_{6}$ and $\mathrm{C}_{6} \mathrm{H}_{10} \mathrm{O}_{5}$ and due to RDA reaction. In addition, the formula was deduced to be $\mathrm{C}_{27} \mathrm{H}_{30} \mathrm{O}_{15} \cdot{ }^{38}$ Then, M32 and M33 were identified based on their $\operatorname{clog} P$ values of -1.22216 and -1.01791 , respectively.

3.4.4. Metabolite M34. M34 was eluted at $8.05 \mathrm{~min}$ with a deprotonated molecular ion $[\mathrm{M}-\mathrm{H}]^{-}$at $m / z$ 415.1517, which was 2 Da less than the value obtained for the parent drug M0. Secondary fragment ions at $m / z 335.0230\left[\mathrm{M}-\mathrm{SO}_{3}-\mathrm{H}\right]^{-}$and $255.0658\left[\mathrm{M}-2 \mathrm{SO}_{3}-\mathrm{H}\right]^{-}$were detected in the $\mathrm{MS} / \mathrm{MS}$ spectrum, suggesting that hydrolyzed LQ had undergone a bis-sulfate conjugation reaction and that the formula was $\mathrm{C}_{15} \mathrm{H}_{12} \mathrm{O}_{10} \mathrm{~S}_{2}$.

3.4.5. Metabolite M35. M35 had a retention time of $8.70 \mathrm{~min}$ with a deprotonated molecular ion $[\mathrm{M}-\mathrm{H}]^{-}$at $\mathrm{m} / \mathrm{z}$ 497.0759, which was 80 Da higher than the value obtained for LQ, suggesting that M35 was a metabolite of sulfate conjugation. Meanwhile, characteristic fragment ions at $\mathrm{m} / \mathrm{z} 417.1185$ $\left[\mathrm{M}-\mathrm{SO}_{3}-\mathrm{H}\right]^{-}, 255.0655\left[\mathrm{M}-\mathrm{SO}_{3}-\mathrm{C}_{6} \mathrm{H}_{10} \mathrm{O}_{5}-\mathrm{H}\right]^{-}$and 135.0074 (RDA reaction) were seen in the $\mathrm{MS} / \mathrm{MS}$ spectrum, implying that the chemical formula was $\mathrm{C}_{21} \mathrm{H}_{22} \mathrm{O}_{12} \mathrm{~S}^{38}$

3.4.6. Metabolite M36. M36 exhibited a deprotonated molecular ion $[\mathrm{M}-\mathrm{H}]^{-}$at $m / z$ 511.0541, which was 94 Da more than the value obtained for deprotonated LQ. In addition a series of important fragment ions were observed at $\mathrm{m} / \mathrm{z}$ $431.0970\left[\mathrm{M}-\mathrm{SO}_{3}-\mathrm{H}\right]^{-}, 255.0654\left[\mathrm{M}-\mathrm{SO}_{3}-\mathrm{C}_{6} \mathrm{H}_{8} \mathrm{O}_{6}-\mathrm{H}\right]^{-}, 135.0084$ ( $\mathrm{RDA}$ reaction) and 119.0506 (RDA reaction) in the MS/MS scan. Moreover, the retention time of $\mathrm{M} 36$ was $8.92 \mathrm{~min}$, and the formula was identified to be $\mathrm{C}_{21} \mathrm{H}_{20} \mathrm{O}_{13} \mathrm{~S}^{38}$

3.4.7. Metabolites M44, M45. The QTOF-MS mass spectra of the M44 and M45 metabolites exhibited deprotonated ions $[\mathrm{M}-\mathrm{H}]^{-}$at $m / z 431.0976$ and 431.0976 , respectively, which were $14 \mathrm{Da}$ higher than the value obtained for the parent drug LQ. In addition, both metabolites exhibited the loss of a glucuronide unit (176 Da) in the MS/MS spectra. A number of typical secondary fragment ions at $m / z$ 255.0658, 135.0086 and
119.0503 were observed due to the loss of $\mathrm{C}_{6} \mathrm{H}_{8} \mathrm{O}_{6}$ and due to RDA reaction. It was evident that hydrolyzed $\mathrm{LQ}$ had undergone glucuronide conjugation, and the molecular formula was identified to be $\mathrm{C}_{21} \mathrm{H}_{20} \mathrm{O}_{10} \cdot{ }^{38} \mathrm{M} 44$ and M45 were eluted at 11.17 and $11.97 \mathrm{~min}$, respectively. The $\operatorname{clog} P$ values of M44 and M45 were 0.27429 and 0.55864 , respectively. Hence, M44 and M45 were identified on the basis of their retention times and $\operatorname{cog} P$ values.

3.4.8. Metabolites M46, M47. The metabolites M46 and M47, eluted at 12.13 and $12.78 \mathrm{~min}$, respectively, were observed in the metabolic profiles with deprotonated molecular ions [M $-\mathrm{H}]^{-}$at $m / z 429.0828$ and 429.0824 , respectively, corresponding to an elemental composition of $\mathrm{C}_{21} \mathrm{H}_{18} \mathrm{O}_{10}$. The masses of the deprotonated M46 and M47 were 12 Da more than the mass of LQ. A characteristic fragment ion at $\mathrm{m} / \mathrm{z} 253.0505$ [M$\left.\mathrm{C}_{6} \mathrm{H}_{8} \mathrm{O}_{6}-\mathrm{H}\right]^{-}$was observed, which confirmed that M46 and M47 were metabolites of glucuronide conjugation. Moreover, M46 and M47 were assigned based on clog $P$ values of 0.390001 and 0.583201 , respectively.

3.4.9. Metabolites M50, M51. M50 and M51 showed deprotonated molecular ions $[\mathrm{M}-\mathrm{H}]^{-}$at $\mathrm{m} / \mathrm{z} 335.0216$ and 335.0217 , respectively, which were 82 Da less than the value obtained for LQ. M50 and M51 were eluted at 12.49 and $17.30 \mathrm{~min}$, respectively. Typical fragment ions at $\mathrm{m} / \mathrm{z} 255.0656$ $\left[\mathrm{M}-\mathrm{SO}_{3}-\mathrm{H}\right]^{-}, 135.0083$ (RDA reaction) and 119.0500 (RDA reaction) were observed in the MS/MS spectra. The neutral loss of $\mathrm{SO}_{3}$ suggested that hydrolyzed LQ had undergone sulfate conjugation, implying that the molecular formula was $\mathrm{C}_{15} \mathrm{H}_{12} \mathrm{O}_{7} \mathrm{~S} .{ }^{38}$ By using ChemDraw 12.0, the clog $P$ values of M50 and M51 were 0.689589 and 0.97394 , respectively. Hence, these metabolites were identified based on the retention times and $\operatorname{cog} P$ values.

3.4.10. Metabolites M53, M54. Two peaks were eluted at 13.24 and 14.22 min with deprotonated molecular ions $[\mathrm{M}-\mathrm{H}]^{-}$ at $m / z 413.0854$ and 413.0853 , respectively, which were 4 Da less 

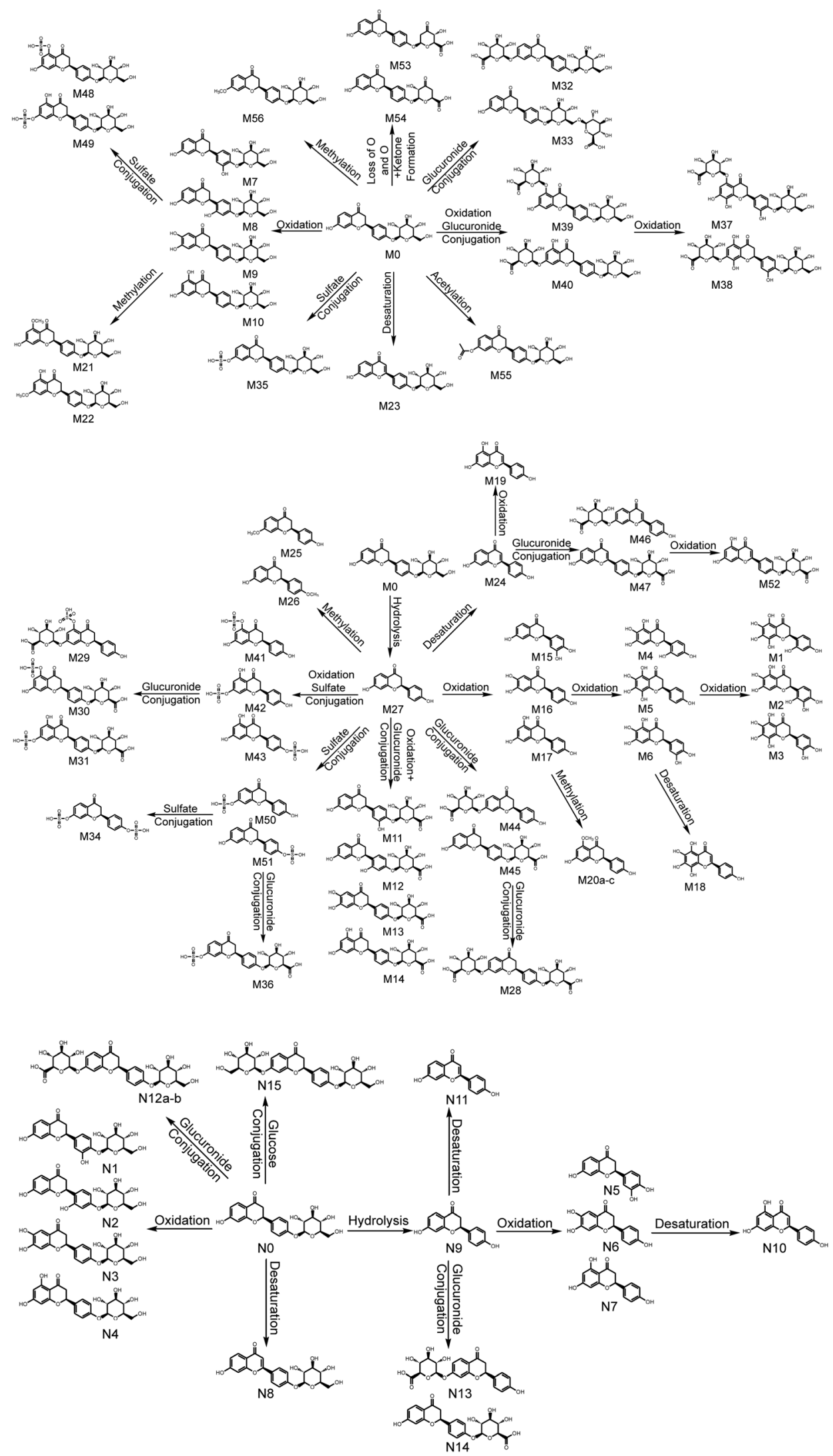

Fig. 5 Metabolic profile and proposed metabolic pathways of $L Q$ in vitro and in vivo ( $a, b, c-$ possible chemical structure). 


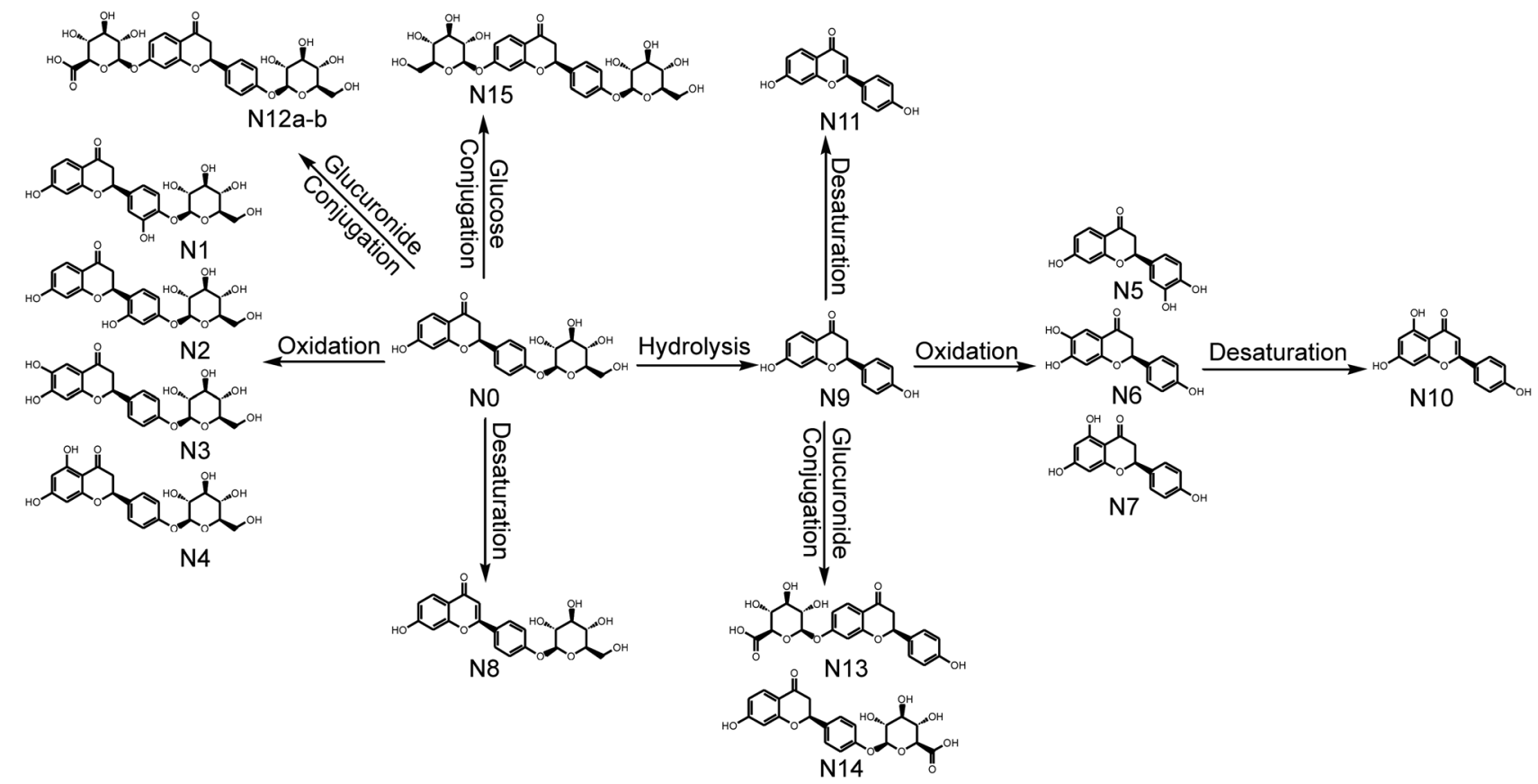

Fig. 5 (Contd.)

than the value obtained for the parent compound LQ. These metabolites had identical fragment ions at $\mathrm{m} / \mathrm{z} 255.0666,135.0087$ and 119.0501. The fragmentation ion at $\mathrm{m} / \mathrm{z} 255.0666$ suggested that the position of oxygen loss was glucose and not flavone. Therefore, the molecular formula was deduced to be $\mathrm{C}_{21} \mathrm{H}_{18} \mathrm{O}_{9}$. In addition, metabolites M53 and M54 were identified based on the $\operatorname{cog} P$ values of 0.29614 and 0.34904 , respectively.

3.4.11. Metabolite M55. M55 was eluted at a retention time of $16.56 \mathrm{~min}$ with a deprotonated molecular ion $[\mathrm{M}-\mathrm{H}]^{-}$at $\mathrm{m} / \mathrm{z}$ 459.1297, which was 42 Da more than the value obtained for LQ. Masses for characteristic ions were observed at $m / z$ 417.1198, $255.0645,135.0070$ and 119.0489 , which were generated by the loss of $\mathrm{CH}_{2} \mathrm{CO}$ and $\mathrm{C}_{6} \mathrm{H}_{10} \mathrm{O}_{5}$ and by RDA reaction, suggesting that M55 was an acetylation metabolite. Moreover, the chemical formula of M55 was $\mathrm{C}_{23} \mathrm{H}_{24} \mathrm{O}_{10}$.

3.4.12. Metabolite M56. M56 eluted at $18.33 \mathrm{~min}$ and had a deprotonated ion $[\mathrm{M}-\mathrm{H}]^{-}$at $m / z 431.0992$, which was $14 \mathrm{Da}$ higher than the value obtained for M0, implying that methylation had occurred. The secondary fragment ions at $m / z 255.0666$ $\left[\mathrm{M}-\mathrm{CH}_{2}-\mathrm{C}_{6} \mathrm{H}_{10} \mathrm{O}_{5}-\mathrm{H}\right]^{-}$and 135.0086 (RDA reaction) demonstrated that the chemical formula was $\mathrm{C}_{22} \mathrm{H}_{24} \mathrm{O}_{9}$. Other metabolites were showed in ESI. $\dagger$

\subsection{Identification and characterization of in vitro metabolites}

In this incubation system, fifteen metabolites were detected, including eleven phase I and four phase II metabolites. Furthermore, metabolites N1-N14 were identified in the in vivo metabolic study. However, N15 was not found in vivo bio-samples. The structures of all the metabolites are shown in Fig. 2.

\subsubsection{Identification of phase I metabolites}

Metabolites N1, N2, M3 and N4. N1-N4, with retention times of $8.62,9.01,9.51$ and $14.03 \mathrm{~min}$, exhibited sharp peaks of deprotonated molecular ions $[\mathrm{M}-\mathrm{H}]^{-}$at $\mathrm{m} / \mathrm{z}$ 433.1110, 433.1111, 433.1112 and 433.1109, respectively, which were 16 Da more than the value obtained for LQ, suggesting that N1$\mathrm{N} 4$ were oxidation metabolites and corresponded to the molecular formula $\mathrm{C}_{21} \mathrm{H}_{22} \mathrm{O}_{10}$. In addition, typical fragment ions at $\mathrm{m} / \mathrm{z} 271.0592,243.0645$ and 109.0289 were generated by the loss of $\mathrm{C}_{6} \mathrm{H}_{10} \mathrm{O}_{5}$ and $\mathrm{CO}$ and by RDA reaction. Moreover, N1N4 were identified based on the $\operatorname{cog} P$ values of N1-N4 at $0.300687,0.500687,0.558399$ and 0.948399 , respectively.

Metabolite N8. N8 was detected at $12.63 \mathrm{~min}$ and exhibited a sharp peak for a deprotonated molecular ion $[\mathrm{M}-\mathrm{H}]^{-}$at $\mathrm{m} / \mathrm{z}$ 415.1011, which was 2 Da less than the value obtained for M0. Fragment ions at $m / z 253.0518\left[\mathrm{M}-\mathrm{C}_{6} \mathrm{H}_{10} \mathrm{O}_{5}-\mathrm{H}\right]^{-}$suggested that a desaturation reaction had occurred, which was consistent with a molecular formula of $\mathrm{C}_{21} \mathrm{H}_{20} \mathrm{O}_{9}$.

Metabolite N9. N9 was detected at $16.03 \mathrm{~min}$. N9 exhibited a sharp peak for a deprotonated molecular ion $[\mathrm{M}-\mathrm{H}]^{-}$at $\mathrm{m} / \mathrm{z}$ 255.0650, which was 162 Da lower than the value obtained for LQ, implying that a hydrolysis had occurred. The secondary fragment ions at $m / z 135.0083$ and 119.0500 , caused by RDA reaction, confirmed that the molecular formula of $\mathrm{N} 9$ was $\mathrm{C}_{15} \mathrm{H}_{12} \mathrm{O}_{4} \cdot{ }^{38}$

Metabolite N10. N10 exhibited a deprotonated molecular ion $[\mathrm{M}-\mathrm{H}]^{-}$at $m / z 269.0437$, which was 14 Da more than the value obtained for N9, indicating that the molecular formula of N10 was $\mathrm{C}_{15} \mathrm{H}_{10} \mathrm{O}_{5}$. In addition, $\mathrm{N} 10$ had a retention time of $16.38 \mathrm{~min}$, and the important fragment ions were at $\mathrm{m} / \mathrm{z}$ 135.0090 (RDA reaction) and 133.0287 (RDA reaction).

Metabolite N11. N11 showed a deprotonated molecular ion [M $-\mathrm{H}]^{-}$at $m / z$ 253.0496, which was 2 Da less than the value obtained for N9, suggesting that a desaturation reaction occurred for N9, and the molecular formula of N11 was predicted to be $\mathrm{C}_{15} \mathrm{H}_{10} \mathrm{O}_{4} \cdot{ }^{38}$ In addition, the secondary fragment ions at $\mathrm{m} / \mathrm{z}$ 
$135.0077,133.0284$ and 117.0341 , caused by RDA reaction, were consistent with the chemical structure of N11. Moreover, the retention time of $\mathrm{N} 11$ was $17.58 \mathrm{~min}$.

\subsubsection{Identification of phase II metabolites}

Metabolite N12. N12 presented a deprotonated molecular ion $[\mathrm{M}-\mathrm{H}]^{-}$at $m / z 593.1565$, which was 176 Da higher than the value obtained for LQ, suggesting that glucuronide conjugation had occurred. In addition, the molecular formula was determined to be $\mathrm{C}_{27} \mathrm{H}_{30} \mathrm{O}_{15}{ }^{38}$ Moreover, the MS/MS spectrum exhibited a series of typical fragment ions at $m / z 417.1208$ [M$\left.\mathrm{C}_{6} \mathrm{H}_{8} \mathrm{O}_{6}-\mathrm{H}\right]^{-}, \quad 255.0659\left[\mathrm{M}-\mathrm{C}_{6} \mathrm{H}_{8} \mathrm{O}_{6}-\mathrm{C}_{6} \mathrm{H}_{10} \mathrm{O}_{5}-\mathrm{H}\right]^{-}, \quad 135.0078$ (RDA reaction) and 117.0187 (RDA reaction). In addition, N12 was eluted at $7.62 \mathrm{~min}$.

Metabolite N15. The molecular formula was determined to be $\mathrm{C}_{27} \mathrm{H}_{32} \mathrm{O}_{14}$ based on the deprotonated molecular ion $[\mathrm{M}-\mathrm{H}]^{-}$ at $\mathrm{m} / z 579.1752$, which was $162 \mathrm{Da}$ higher than the value obtained for LQ. N15 was eluted at $12.45 \mathrm{~min}$ and had characteristic fragment ions at $\mathrm{m} / \mathrm{z} 417.1273\left[\mathrm{M}-\mathrm{C}_{6} \mathrm{H}_{10} \mathrm{O}_{5}-\mathrm{H}\right]^{-}, 255.0669$ $\left[\mathrm{M}-2 \mathrm{C}_{6} \mathrm{H}_{10} \mathrm{O}_{5}-\mathrm{H}\right]^{-}, 135.0085$ (RDA reaction) and 119.0492 (RDA reaction), which implied that N15 was a glucose metabolite. Other metabolites were showed in ESI. $\dagger$

\subsection{Metabolic pathways of LQ}

Based on the elemental compositions of the metabolites, the accurate MS/MS spectra, the chemical structures of the metabolites and the fragment ions of the metabolites, the metabolic pathway of LQ could be tentatively proposed. In total, 56 in vivo metabolites and 15 in vitro metabolites were characterized. Based on these results, LQ mainly underwent oxidation, reduction, hydrolysis, methylation, acetylation, glucuronide and sulfate conjugation. Based on the in vivo metabolic data, sulfate and glucuronide conjugation reactions were the major bio-transformations. However, based on the in vitro metabolic data, the metabolic pathways of LQ were concentrated in phase I. The proposed metabolic pathways of LQ in vitro and in vivo are shown in Fig. 5.

\subsection{Comparison of metabolic pathways in vitro and in vivo}

Drug metabolism plays an important role in different areas of the pharmaceutical industry and in drug development and toxicology. The in vivo approach is quantitative and very effective in studies of drug metabolism. ${ }^{\mathbf{4 9 , 5 0}}$ The in vitro method is generally suitable for targeted studies and is often predictive of real hazards and risks. ${ }^{51}$ In this study, in vitro (rat liver microsomes) and in vivo (blood, urine, feces and bile in rats) metabolic profiles were investigated by UHPLC-Q-TOF-MS/MS. Consequently, a total of 56 in vivo metabolites and 15 in vitro metabolites were screened and characterized. The in vitro and in vivo metabolisms were concentrated in phase I and II, respectively. The metabolites detected in vitro were all detected in vivo as well. However, the isomer of N12 was detected in vivo, and N15 was not found in vivo. It was speculated that oxidation of N15 had occurred in vivo. Therefore, the isomer of N12 was observed in vivo, which suggested that the metabolism in vivo was more complex and elusive than that in vitro.

\subsection{Comparison of metabolites identified in this study and} in a previous study

A study of the metabolic pathways of LQ has been reported in China, which only detected 7 metabolites (M24, M27, M32 (N12), M35, M36, M44 and M45 (N13 and N14), and M51). However, in this study, 56 metabolites were detected in vivo and 15 metabolites were detected in vitro, including the 7 metabolites identified in the previous study. Moreover, the metabolic sites of two metabolites were not determined in the previous study, and there were some differences between this study and the previous study. In this manuscript, M32, M33 and M50, M51 were detected as two pairs of isomers; however, these metabolites were detected as a single chromatographic peak in the previous study. Moreover, in this study, M32 and M33 were both not detected in vitro (N12), and no isomers of M36 were detected in vivo. Furthermore, in this manuscript, the metabolic sites of M44 and M45 were identified based on the important $\operatorname{clog} P$ values, which were the same as those of N13 and N14 in vitro. In addition, in this study, metabolite N15 was not detected in vivo; therefore, it was speculated that N15 had undergone oxidation and that metabolites M32 and M33 were found in vivo.

\section{Conclusion}

In conclusion, the UHPLC-Q-TOF-MS/MS method was first established to screen and identify the metabolites of LQ in vitro and in vivo. Based on the preceding data acquisition and mining strategy, twenty, fifty-four, forty-eight, thirty-seven and fifteen metabolites were detected in rat blood, urine, bile, feces and liver microsomes, respectively. The metabolic pathways of LQ were determined to be oxidation, reduction, hydrolysis, methylation, acetylation, and sulfate and glucuronide conjugation; these findings filled in the gaps remaining from the previous incomplete study. Key product ion (KPI) was used to aid the detection of metabolites of LQ. The results lay the foundation for active screening studies. In addition, this study demonstrated a powerful strategy for rapid screening and identifying metabolites and chemical constituents of traditional Chinese medicines.

\section{Conflicts of interest}

All the authors have declared no conflict of interest.

\section{Acknowledgements}

The work received financial support from the National Natural Science Foundation of China (No. 81473180).

\section{References}

1 M. N. Asl and H. Hosseinzadeh, Phytother. Res., 2008, 22, 709-724.

2 B. Q. Fu, H. Li, X. R. Wang, F. S. C. Lee and S. F. Cui, J. Agric. Food Chem., 2005, 53, 7408-7414. 
3 J. Cinatl, B. Morgenstern, G. Bauer, P. Chandra, H. Rabenau and H. W. Doerr, Lancet, 2003, 361, 2045-2046.

4 T. Yokozawa, Z. W. Liu and C. P. Chen, Phytomedicine, 2000, 6, 439-445.

5 J. He, L. Chen, D. Heber, W. Y. Shi and Q. Y. Lu, J. Nat. Prod., 2006, 69, 121-124.

6 G. Khaksa, M. E. Zolfaghari, A. R. Dehpour and T. Samadian, Planta Med., 1996, 62, 326-328.

7 F. Rauchensteiner, Y. Matsumura, Y. Yamamoto, S. Yamaji and T. Tani, J. Pharm. Biomed. Anal., 2005, 38, 594-600.

8 Y. X. Gao, B. F. Cheng, J. J. Lian, D. D. Guo, J. W. Qin, Y. B. Zhang, H. J. Yang, M. Wang, L. Wang and Z. W. Feng, J. Funct. Foods, 2017, 33, 142-148.

9 H. J. Kwon, H. H. Kim, Y. B. Ryu, J. H. Kim, H. J. Jeong, S. W. Lee, J. S. Chang, K. O. Cho, M. C. Rho, S. J. Park and W. S. Lee, Bioorg. Med. Chem., 2010, 18, 7668-7674.

10 P. Montoro, M. Maldini, M. Russo, S. Postorino, S. Piacente and C. Pizza, J. Pharm. Biomed. Anal., 2011, 54, 535-544.

11 X. Y. Meng, S. B. Yang, Z. F. Pi, F. R. Song, H. Y. Jiang and Z. Q. Liu, J. Liq. Chromatogr. Relat. Technol., 2012, 35, 1538-1549.

12 C. F. Xue, S. Jiang, J. M. Guo, D. W. Qian, J. A. Duan and E. X. Shang, J. Chromatogr. B: Anal. Technol. Biomed. Life Sci., 2011, 879, 3901-3908.

13 C. Y. Li, L. W. Qi and P. Li, J. Pharm. Biomed. Anal., 2011, 55, 146-160.

14 G. G. Tan, M. Liu, X. Dong, S. Wu, L. Fan, Y. B. Qiao, Y. F. Chai and H. Wu, J. Pharm. Biomed. Anal., 2014, 96, 187-196.

15 Y. Z. Zhang, F. Xu, J. Dong, J. Liang, Y. Hashi, M. Y. Shang, D. H. Yang, X. Wang and S. Q. Cai, J. Pharm. Biomed. Anal., 2012, 70, 425-439.

16 M. P. Gonthier, C. Remesy, A. Scalbert, V. Cheynier, J. M. Souquet, K. Poutanen and A. M. Aura, Biomed. Pharmacother., 2006, 60, 536-540.

17 A. H. Yang, J. X. Chen, Y. T. Ma, L. L. Wang, Y. W. Fan and X. He, J. Pharm. Biomed. Anal., 2017, 141, 200-209.

18 M. Y. Liu, S. H. Zhao, Z. Q. Wang, Y. F. Wang, T. Liu, S. Li, C. C. Wang, H. T. Wang and P. F. Tu, J. Chromatogr. B: Anal. Technol. Biomed. Life Sci., 2014, 949-950, 115-126.

19 H. Han, W. L. Zeng, C. Y. He, S. W. A. Bligh, Q. Liu, L. Yang and Z. T. Wang, J. Mass Spectrom., 2014, 49, 1108-1116.

20 K. Wang, L. W. Chai, X. C. Feng, Z. B. Liu, H. X. Liu, L. Q. Ding and F. Qiu, J. Pharm. Biomed. Anal., 2017, 139, 73-86.

21 X. D. Cheng and M. G. Wei, Molecules, 2014, 19, 1888118896.

22 D. G. Wang, T. J. Hang, C. Y. Wu and W. Y. Liu, J. Chromatogr. B: Anal. Technol. Biomed. Life Sci., 2005, 829, 97-106.

23 H. L. Ma, Y. Liu, X. Mai, Y. J. Liao, K. Zhang, B. Liu, X. Xie and Q. L. Du, J. Pharm. Biomed. Anal., 2016, 125, 194-204.

24 K. Xiong, T. T. Gao, T. Zhang, Z. T. Wang and H. Han, J. Chromatogr. B: Anal. Technol. Biomed. Life Sci., 2017, 10651066, 1-7.

25 C. P. Yang, M. H. Liu, W. Zou, X. L. Guan, L. Lai and W. W. Su, J. Asian Nat. Prod. Res., 2012, 14, 68-75.
26 C. R. Deng, C. Y. Gao, X. H. Tian, B. Chao, F. Wang, Y. Zhang, J. T. Zou and D. C. Liu, J. Funct. Foods, 2017, 35, 332-340.

27 Y. Y. Zhao, S. P. Wu, S. M. Liu, Y. M. Zhang and R. C. Lin, Chem.-Biol. Interact., 2014, 220, 181-192.

28 Y. Y. Zhao and R. C. Lin, Chem.-Biol. Interact., 2014, 215, 716.

29 Y. Y. Zhao, L. Zhang, F. Y. Long, X. L. Cheng, X. Bai, F. Wei and R. C. Lin, Chem.-Biol. Interact., 2013, 201, 31-38.

30 Y. Y. Zhao, J. Liu, X. L. Cheng, X. Bai and R. C. Lin, Clin. Chim. Acta, 2012, 413, 642-649.

31 X. Wang, S. S. Johansen, M. K. K. Nielsen and K. Linnet, Drug Test. Anal., 2017, 9, 1137-1151.

32 J. M. Wang, Z. Yang and J. Lechago, Biomed. Chromatogr., 2013, 27, 1463-1480.

33 S. Hegstad, S. Hermansson, I. Betner, O. Spigset and B. M. H. Falch, J. Chromatogr. B, 2014, 947-948, 83-95.

34 J. J. Pitt, Clin. Biochem. Rev., 2009, 30, 19-34.

35 A. H. Wu, R. Gerona, P. Armenian, D. French, M. Petrie and K. L. Lynch, Clin. Toxicol., 2012, 50, 733-742.

36 H. H. Maurer, Ther. Drug Monit., 2010, 32, 324-327.

37 H. H. Maurer and M. R. Meyer, Arch. Toxicol., 2016, 90, 21612172.

38 S. Q. Dong, H. R. Fan, Q. S. Li, G. L. Wei, W. H. Liu and D. Y. Si, Chin. Tradit. Herbal Drugs, 2014, 45, 2499-2505.

39 W. T. Liang, H. B. Huang and T. Liu, Pharm. Today, 2012, 22, 13-16.

40 H. B. Li, Z. R. Li, Z. L. Wang and L. Y. Sun, Prog. Biochem. Biophys., 1993, 20, 402-403.

41 Y. H. Ma, W. W. Xie, T. T. Tian, Y. R. Jin, H. J. Xu, K. R. Zhang and Y. F. Du, Anal. Biochem., 2016, 511, 61-73.

42 P. P. Jia, Y. Q. Zhang, Q. Y. Zhang, Y. P. Sun, H. T. Yang, H. Shi, X. X. Zhang and L. T. Zhang, Biomed. Chromatogr., 2016, 30, 1498-1505.

43 M. Y. Liu, S. H. Zhao, Z. Q. Wang, Y. F. Wang, T. Liu, S. Li, C. C. Wang, H. T. Wang and P. F. Tu, J. Chromatogr. B: Anal. Technol. Biomed. Life Sci., 2014, 949-950, 115-126.

44 T. T. Tian, Y. R. Jin, Y. H. Ma, W. W. Xie, H. J. Xu, K. R. Zhang, L. T. Zhang and Y. F. Du, J. Chromatogr. B: Anal. Technol. Biomed. Life Sci., 2015, 1006, 80-92.

45 J. Liang, F. Xu, Y. Z. Zhang, S. Huang, X. Y. Zang, X. Zhao, L. Zhang, M. Y. Shang, D. H. Yang, X. Wang and S. Q. Cai, J. Pharm. Biomed. Anal., 2013, 83, 108-121.

46 J. F. Chen, Y. L. Song, X. Y. Guo, P. F. Tu and Y. Jiang, Analyst, 2014, 139, 6474-6485.

47 L. L. Wang, M. M. Sang, E. W. Liu, P. O. Banahene, Y. Zhang, T. Wang, L. F. Han and X. M. Gao, J. Pharm. Biomed. Anal., 2017, 140, 45-61.

48 K. W. Luo, Q. S. Shi and F. Feng, J. Chromatogr. B: Anal. Technol. Biomed. Life Sci., 2017, 1040, 260-272.

49 S. J. Fenwick and J. P. Scarth, Drug Test. Anal., 2011, 3, 705716.

50 J. P. Scarth, H. A. Spencer, S. E. Timbers, S. C. Hudson and L. L. Hillyer, Drug Test. Anal., 2010, 2, 1-10.

51 I. Wilk-Zasadna, C. Bernasconi, O. Pelkonen and S. Coecke, Toxicology, 2015, 332, 8-19. 